\title{
The use of joint mobilization on mechanical instability deficits for a lateral ankle sprain: A Systematic Review
}

Kathleen M. Kerecman

West Virginia University

Follow this and additional works at: https://researchrepository.wvu.edu/etd

\section{Recommended Citation}

Kerecman, Kathleen M., "The use of joint mobilization on mechanical instability deficits for a lateral ankle sprain: A Systematic Review" (2014). Graduate Theses, Dissertations, and Problem Reports. 554.

https://researchrepository.wvu.edu/etd/554

This Thesis is protected by copyright and/or related rights. It has been brought to you by the The Research Repository @ WVU with permission from the rights-holder(s). You are free to use this Thesis in any way that is permitted by the copyright and related rights legislation that applies to your use. For other uses you must obtain permission from the rights-holder(s) directly, unless additional rights are indicated by a Creative Commons license in the record and/ or on the work itself. This Thesis has been accepted for inclusion in WVU Graduate Theses, Dissertations, and Problem Reports collection by an authorized administrator of The Research Repository @ WVU. For more information, please contact researchrepository@mail.wvu.edu. 
The use of joint mobilization on mechanical instability deficits for a lateral ankle sprain:

A Systematic Review

Kathleen M. Kerecman, BS, ATC

Thesis submitted to the

College of Physical Activity and Sport Sciences

at West Virginia University

in partial fulfillment of requirements

for the degree of

Master of Science

in

Athletic Training

Michelle A. Sandrey, Ph.D., ATC, Chair

Patricia Riley, ATC

Jean McCrory, Ph.D

Department of Sport Sciences

Morgantown, WV

2014

Key Words: lateral ankle sprain, joint mobilizations, ankle, treatment 


\section{ABSTRACT}

The use of joint mobilization on mechanical instability deficits for a lateral ankle sprain:

\section{A Systematic Review}

\section{Kathleen M. Kerecman}

Context: The use of manual therapy in the athletic training setting is becoming more common place. While there is information on joint mobilizations, there are few systematic reviews that examine the use of joint mobilizations on mechanical deficits in chronic ankle instability and lateral ankle sprain. Objective: To understand the benefits of joint mobilization in the lateral ankle sprain (chronic and acute) and determine common parameters/techniques used in practice. Data Sources: Pub Med (1950-2013), CINAHL with full text (1982-2013), MEDLINE (1950-2013), SPORTDiscus with full text (18002013), Science Direct(1950-2013), Google Scholar (1950-2013), and PEDro (1929-2013) were searched using the terms lateral ankle sprain, chronic ankle instability (CAI), and joint mobilization. Next lateral ankle sprain and CAI were searched with the following: Maitland mobilization, Mulligan's mobilization, mobilization with movement, manual therapy, and treatment. Lastly citations were searched for possible references not previously found. Study Selection: Studies were included if they are: 1) Written or translated into the English language, 2) Lateral Ankle sprain, chronic ankle instability, or joint mobilizations were included in the title or subject of the study, 3) Lateral ankle sprain, chronic ankle instability, or joint mobilizations were included in the abstract, 4) The main focus of the study must be joint mobilizations, 5) The study must be an experimental study. Data Extraction: All studies that met the inclusion criteria were examined using the PEDro Scale and a comparison of effect sizes. Each study was read without the use of the PEDro Scale and then again with the use of the PEDro Scale. Scores were then compared between investigators. If differences in scores occurred, it was discussed until an agreement of final score was reached. Data Synthesis: There was a total of 16 studies that were analyzed for this review. They varied with subject populations, mobilization type, exclusion and inclusion criteria, methodological quality, and effect sizes. The PEDro scores ranged from 1 to 8 with an average score of 5.81. Effect sizes were calculated in 11 of the 16 studies. Only 4 of these studies had effect sizes that did not cross 0 and were large. Conclusion: Overall, the studies evaluated had poor methodological quality. While most studies did show an improvement in dorsiflexion ROM, posterior talar glide, pain levels, and H/M ratios, recommendations for the clinician cannot be based off of these studies due to poor methodological quality and effect sizes that encompass 0 . More studies should be conducted to improve statistical significance and methodological quality to improve the current evidence. 


\section{ACKNOWLEDGEMENTS}

To my family who have supported me throughout the years and have always encouraged me to be dedicated to my work and education.

To Mike Casselman for helping to enhance my skills and build my confidence as an entry level athletic trainer.

To all the staff at HealthWorks for helping to teach me new clinical skills that can benefit my patients in future years.

To Dr. Damien Clement for taking the time to score the many articles found in this study. The quickness in which you scored these articles to meet my deadlines was very much appreciated.

To both Patti Riley and Dr. Jean McCrory for being on my thesis committee. Thank you for putting in the time to better my thesis and allowing me to gain a valuable understanding of joint mobilizations.

To all those involved in my athletic training education both here at West Virginia University and at Kent State University. All of the knowledge and practical experiences I have gained have helped me to become the athletic trainer I am today.

To my friends, classmates, and co-workers for their support and understanding throughout the stressful times over the last two years.

And finally to Dr. Sandrey for her guidance and instruction throughout the whole thesis process as well as the past two years. 


\section{TABLE OF CONTENTS}

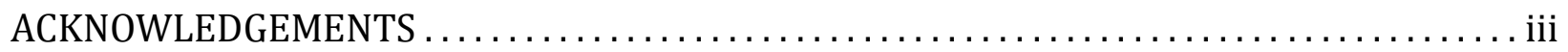

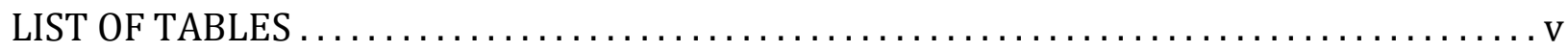

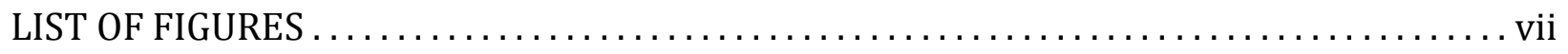

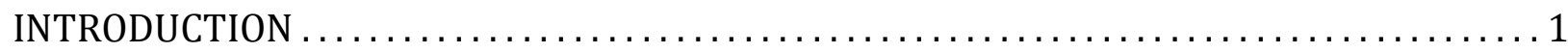

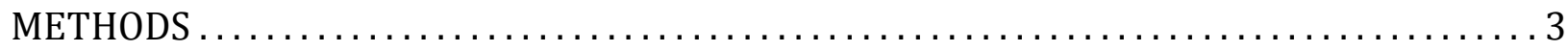

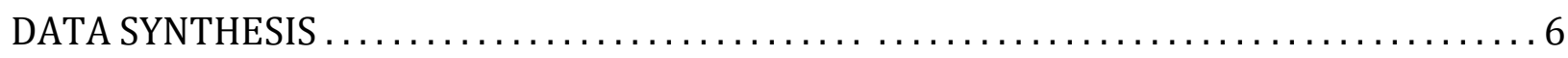

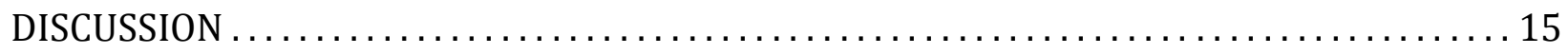

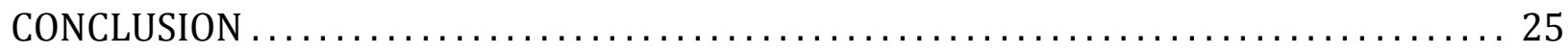

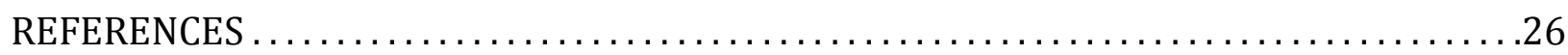

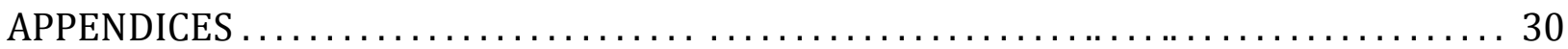

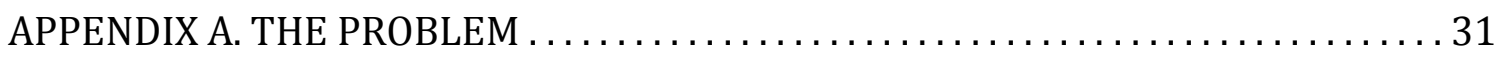

APPENDIX B. LITERATURE REVIEW $\ldots \ldots \ldots \ldots \ldots \ldots \ldots \ldots \ldots \ldots \ldots \ldots \ldots, 37$

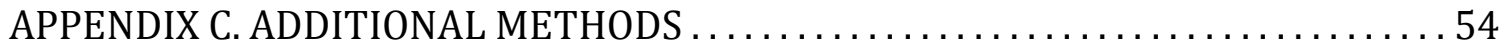

APPENDIX D. ADDITIONAL RESULTS $\ldots \ldots \ldots \ldots \ldots \ldots \ldots \ldots \ldots \ldots \ldots \ldots \ldots$

APPENDIX E. RECCOMENDATIONS FOR FUTURE RESEARCH $\ldots \ldots \ldots \ldots \ldots \ldots 86$

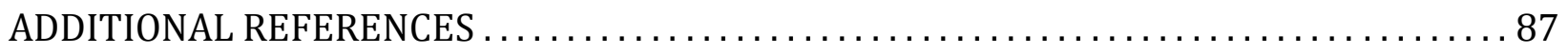




\section{LIST OF TABLES}

Table

Page

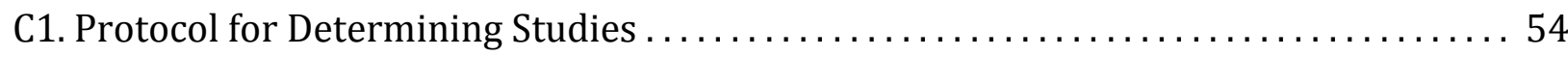

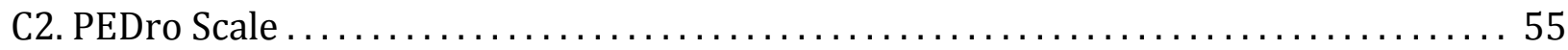

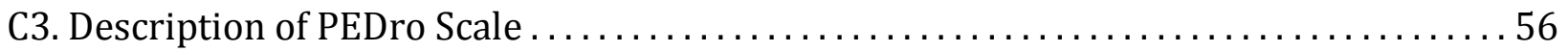

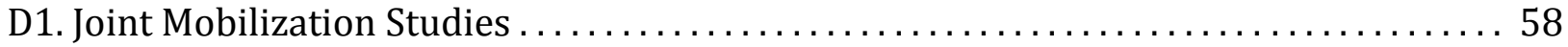

D2. Mobilization with Movement Studies .............................. 59

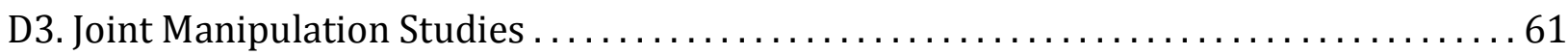

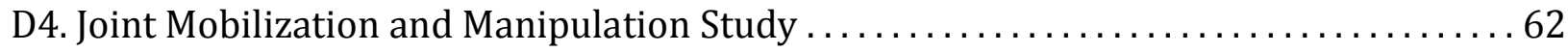

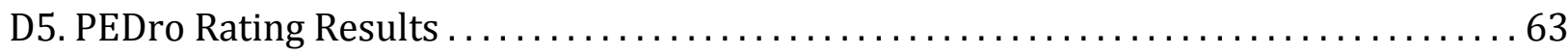

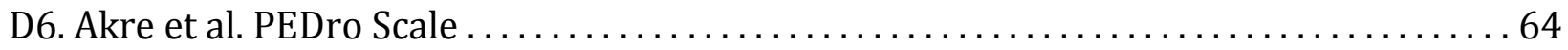

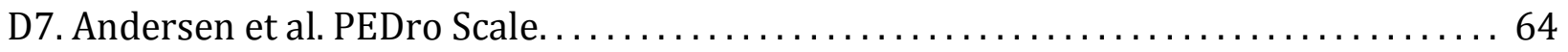

D8. Beazell et al. PEDro Scale. ....................................... 65

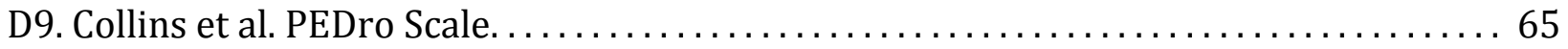

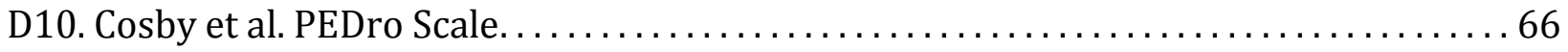

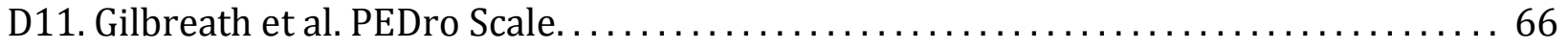

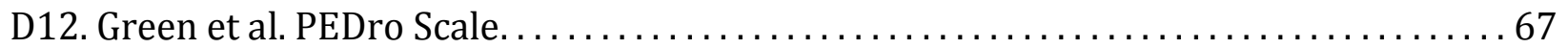

D13. Grindstaff et al. PEDro Scale.................................... 67

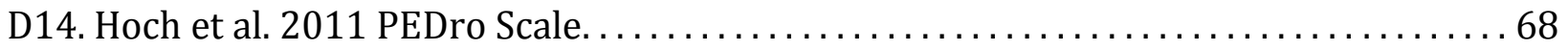

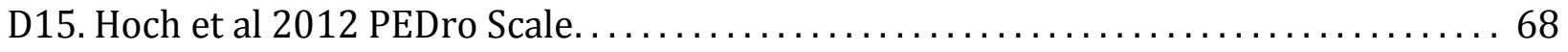

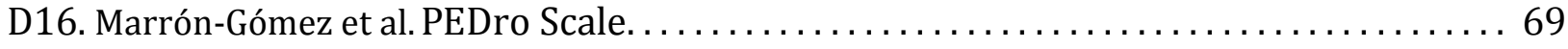

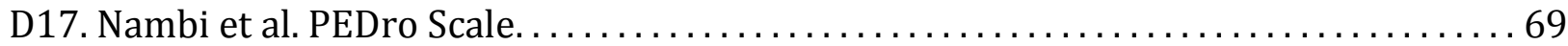

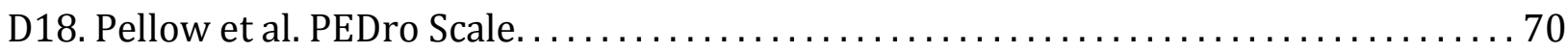

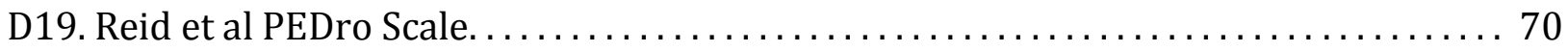

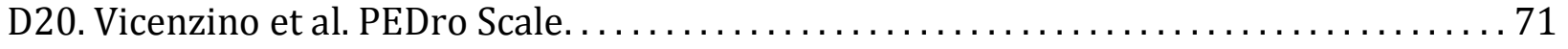




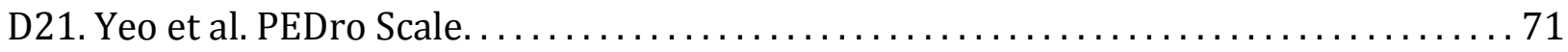

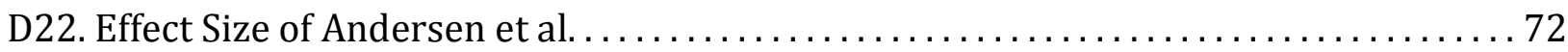

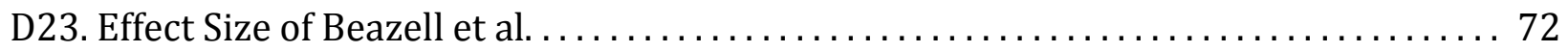

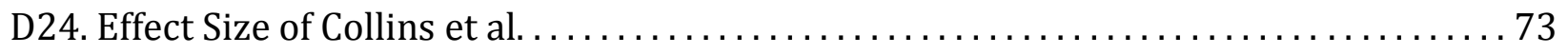

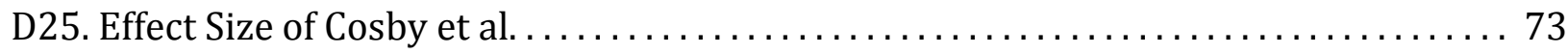

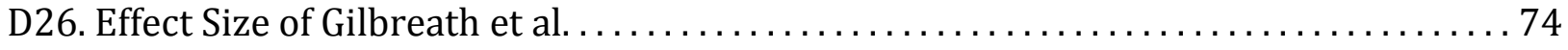

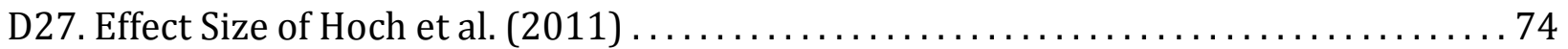

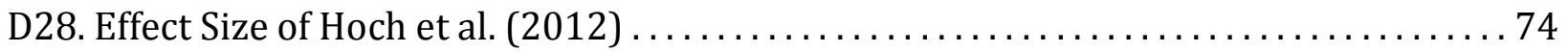

D29. Effect Size of Marrón-Gómez et al. $\ldots \ldots \ldots \ldots \ldots \ldots \ldots \ldots \ldots \ldots \ldots \ldots \ldots \ldots \ldots \ldots \ldots$

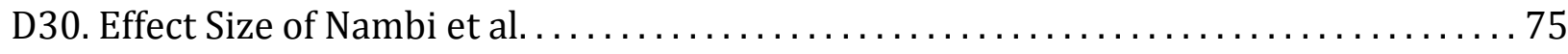

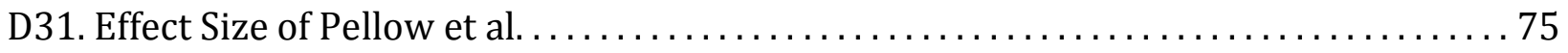

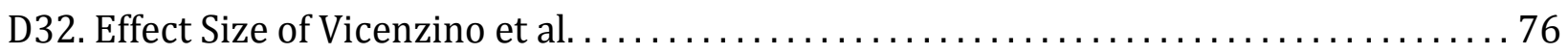




\section{LIST OF FIGURES}

Figure Page

D1. Figure of Studies Found: PubMed $\ldots \ldots \ldots \ldots \ldots \ldots \ldots \ldots \ldots \ldots \ldots \ldots \ldots \ldots \ldots \ldots$

D2. Figure of Studies Found: CINAHL $\ldots \ldots \ldots \ldots \ldots \ldots \ldots \ldots \ldots \ldots \ldots \ldots \ldots \ldots \ldots \ldots$

D3. Figure of Studies Found: MEDLINE $\ldots \ldots \ldots \ldots \ldots \ldots \ldots \ldots \ldots \ldots \ldots \ldots \ldots \ldots \ldots$

D4. Figure of Studies Found: SPORTDiscus $\ldots \ldots \ldots \ldots \ldots \ldots \ldots \ldots \ldots \ldots \ldots \ldots \ldots \ldots \ldots \ldots \ldots$

D5. Figure of Studies Found: Science Direct $\ldots \ldots \ldots \ldots \ldots \ldots \ldots \ldots \ldots \ldots \ldots \ldots \ldots \ldots 2$

D6. Figure of Studies Found: Google Scholar $\ldots \ldots \ldots \ldots \ldots \ldots \ldots \ldots \ldots \ldots \ldots \ldots \ldots$

D7. Figure of Studies Found: PEDro Database $\ldots \ldots \ldots \ldots \ldots \ldots \ldots \ldots \ldots \ldots \ldots \ldots$

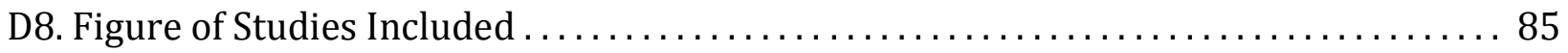




\section{INTRODUCTION}

Lateral ankle sprains are a common injury to both the athletic and sedentary population. In the United States there is an injury rate of 2.15 per 1000 person-years. ${ }^{1}$ About half of the injures reported were due to athletic injury. ${ }^{1}$ An injury rate of 33.89 injuries per 1000 person-years was found in all 4 branches of the United States military. ${ }^{2}$ This is important to note because the United States military has a high level of physical activity, which puts them at a greater risk of injury, similar to an athletic population. In England injury rates of a professional soccer team and youth soccer academies were reported. A total of 8 ankle injuries per 1000 hours of exposure were found in the professional soccer level over the course of 7 years. ${ }^{3}$ There was a median of 0.342 ankle injuries per 1000 hours of athletic exposure. ${ }^{4}$ Athletes from the high school and college setting were found to have a first time injury rate of 0.85 per 1000 person-days of sport exposure. ${ }^{5}$ Based on the prevalence reported, the incidence of ankle injuries in the athletic population is mostly higher than the general population.

Other factors also influence injury rate other than athletic activity. It has been found that injury rate is higher in competition than in practice. ${ }^{4}$ This is because athletes tend to play harder and take greater risks when in competition compared to practice. Injury rates are also at the highest in the 15-23 age group. 1,2,4 Women also tend to have a higher incidence of injury than men in most cases. 1, 2, 5,6

Lateral ankle sprains are typically caused by an inversion force to the foot causing stress in the ankle. ${ }^{7-11}$ This stress often causes a stretching or tearing in the anterior talofibular ligament (ATFL) or the calcaneofibular ligament (CFL). ${ }^{7-11}$ Signs and symptoms of an ankle sprain typically include pain, decreased range of motion (ROM), decreased 
strength, increased joint laxity, and increased swelling. ${ }^{7-11}$ Once damaged ligamentous laxity is present in the ankle even at 8 weeks post-injury. This laxity can cause anatomical and biomechanical compensations that may predispose the patient to more lateral ankle sprains. ${ }^{12}$ This lingering laxity in addition to altered biomechanics could be a reason why ankle sprains have a high risk of re-injury.

Often multiple ankle sprains can lead to chronic ankle instability. Chronic ankle instability (CAI) is thought to be caused by a combination of both functional and mechanical instability. 7,8 Functional instability occurs when balance and proprioception are disrupted. ${ }^{7,8}$ Mechanical instability occurs when there is an anatomic change such as joint laxity, impaired arthrokinematics, and synovial inflammation. ${ }^{7,8}$ The anatomical changes noted are often an anteriorly displaced fibula, ${ }^{8,13}$ laxity in ATFL and CFL, ${ }^{7-8,11,13}$ and reduced posterior glide. ${ }^{14}$ These changes can alter the ankle biomechanics by allowing greater frontal plane displacement and maximum eversion, as well as decreased sagittal plane displacement and maximum plantarflexion during functional tasks. ${ }^{15}$ Commonly a decrease in dorsiflexion ROM has also been noted with chronic ankle sprains. ${ }^{11,13,14}$ Both of these components lead to CAI and prevent joint stability from occurring.

Treatment for ankle sprains often includes a period of rest, increasing ROM, increasing strength, decreasing pain, and increasing proprioception. ${ }^{11,16}$ During this time various therapeutic exercises with the addition of ice and compression are used. ${ }^{16}$ Often in the lateral ankle sprain dorsiflexion ROM is the hardest to gain and can cause a biomechanical pre-disposition for increased ankle sprains. ${ }^{8,9}$ Thus, manual therapy techniques can also be used to assist with ankle healing after an ankle sprain. Techniques including lymphatic drainage, joint mobilizations, and stretching techniques are used. ${ }^{9}$ 
Joint mobilizations are often very effective in increasing ROM, proprioception, balance, and decreasing pain levels. ${ }^{9}, 11,17,18$, Joint mobilizations can help to increase the available ROM at the ankle and correct the mechanical changes that have been altered to cause a change in function and biomechanics. With the addition of normal ankle management joint mobilizations can be used to help return mechanical changes closer to pre-injury levels and return the athlete to competition faster and more effectively.

There have been multiple studies $9,19,20$ that have reviewed joint mobilizations and effectiveness. Most reviews have evaluated treatment to correct functional instabilities in the lateral ankle. Very few have considered the mechanical instabilities and treatment using joint mobilizations, Maitland mobilizations, and Mulligan's mobilizations (also called mobilizations with movement or MWM) in regard to their application on the lateral ankle sprain and chronic ankle instability. Thus, the purpose of this study is to evaluate the

literature on the use of joint mobilizations to decrease mechanical instabilities in the lateral ankle sprain. Each study will be evaluated by the PEDro scale and by effect sizes. This study aims to focus on joint mobilizations at the ankle specifically to evaluate the benefits of treatment, the effectiveness of causing those benefits, and if different joint mobilization types have a difference in these outcomes.

\section{METHODS}

Design

This study is a systematic review. Studies were found through searches of databases using keywords, combination of keywords, or cross referencing. Studies were then evaluated by the PEDro scale and the effect sizes were compared. A systematic review 
is an effective way to gather information and evaluate the methodological quality of the study to determine the effectiveness of the treatment.

Instrumentation

The Physiotherapy Evidence Database (PEDro) scale is an 11 item scale that was created to rate the methodological quality of randomized controlled studies. ${ }^{21,22}$ The PEDro scale measures only the methodological quality of clinical trials and has been growing in use over the past several years. ${ }^{23}$ Each item contributes to one point of the score except for the first item. The items are based from the Delphi consensus technique and the Jadad scale. ${ }^{21}$ The reliability rating of PEDro scale items varied from "fair to substantial" and the reliability for the total PEDro score was found to be "fair to good". ${ }^{21}$ The PEDro scale has a high validity to be used as an indicator of methodological quality when compared to Rasch analysis. ${ }^{24}$ In the samples, examined hierarchy item adherence from least to most adhered too were identical. The studies adhere to therapist blinding, subject blinding, intention-to-treat analysis, concealed allocation, assessor blinding, less than $85 \%$ follow up, similarity at baseline, point and variability measures, between-group statistical comparison, and random allocation in this order. ${ }^{24}$ In this review, only the PEDro scale was used on physical therapy studies. More research would be needed to determine if it was valid for other disciplines. Full explanation of the PEDro scale can be found in Table C2 and C3.

Data Sources

Pub Med (1950-2013), CINAHL with full text (1982-2013), MEDLINE (1950-2013), SPORTDiscus with full text (1800-2013), Science Direct(1950-2013), Google Scholar 
(1950-2013), and PEDro (1929-2013) databases were searched. Key terms used were lateral ankle sprain, chronic ankle instability, and joint mobilization. Next lateral ankle sprain and CAI were searched with the following: Maitland mobilizations, Mulligan's mobilization, mobilization with movement, manual therapy, and treatment. Lastly citations were searched for possible references not previously found. All studies were written or translated into English and were peer reviewed studies. Studies were taken and compared to inclusion and exclusion criteria. Once studies were accepted into this study they were evaluated using the PEDro scale and effect sizes were calculated if possible.

\section{Study Selection}

Inclusion criteria for study selection included the following: 1) studies were written or translated into English; 2) lateral ankle sprain, chronic ankle instability, or joint mobilizations were present in the title or subject of the study; 3) lateral ankle sprain, chronic ankle instability, or joint mobilization were included in the abstract; 4) the main focus of the study must be joint mobilizations; and 5) studies must be an experimental study. Exclusion criteria for study selection included: 1) studies that were not translated or written in English; 2) studies that focused on functional instability outcome measures (ie. balance or proprioception); and 3) studies that focused on other body parts other than the ankle.

\section{Data Extraction}

Both the PEDro scale and effect size were used as the data extraction for this review. The PEDro scale was used to evaluate the methodological quality. The effect size was evaluated by using Cohen's d. Two investigators were trained on the use of the PEDro scale 
through the PEDro website. After training was completed studies that met inclusion criteria were evaluated. Studies were read twice, once without the PEDro scale and then with the use of the PEDro scale. Each study was given a score from 0 to 10 based on the checklist. If a discrepancy occurred between investigators, the scores were discussed until a final score was agreed upon. The PEDro scale has been shown to have a high reliability when used with systematic reviews. ${ }^{21}$

The effect size estimate was used to examine the results of the study. This was done to determine if the outcomes could be applied to a general population and if this could be considered a valid form of treatment. Cohen's d effect size was calculated taking the mean difference of experimental and control group and dividing it by the standard deviation of the control group. ${ }^{25}$ Upper and lower confidence intervals (CI) were calculated by multiplying the standard error by Z-value for $95 \% \mathrm{CI}^{25}$ The standard deviation is then subtracted or added to this value. Cohen's d uses a standard scale where less than 0.4 is a weak effect, between 0.41 and 0.7 is a moderate effect, and greater than 0.7 is strong. ${ }^{25}$ This study examined the effect that joint mobilization has on range of motion, pain levels, and mechanical outcomes. The effect size comparisons measured the differences between pre-test and post-test or compared control group and experimental group for each study and outcome.

\section{DATA SYNTHESIS}

Study Quality

All sixteen studies that met the inclusion criteria were evaluated with the PEDro scale. ${ }^{26-41}$ These studies had varying subject populations, study locations and individual inclusion/exclusion criteria. A brief summary of study design and results can be found in 
Table D1-D4. The study populations consisted of individuals who had at least one ankle sprain and some reporting chronic ankle instability. Fifteen studies listed inclusion and exclusion criteria. ${ }^{26,28-41}$ Only the Andersen et al. ${ }^{27}$ study did not list inclusion and exclusion criteria. Inclusion criteria consisted of an age range, $26,30,37,38$ ankle sprain $26,29,32,38,41$ or instability, ${ }^{28,31,33-36,40}$ willingness to participate in the study, ${ }^{26,28}$ dorsiflexion ROM deficit compared to opposite side, $28-30,33,39-41$ tenderness over lateral ankle, 29, 32, 37, 41 full pain-free WB capacity, ${ }^{29}$ immobilized in a walking boot, ${ }^{30}$ physically active, ${ }^{31}$ and reported function loss. ${ }^{34-36,41}$ Exclusion criteria consisted of previous history of lower extremity injury other than lateral ankle sprain, ${ }^{26,28-41}$ previous experience or knowledge of MWM, ${ }^{26,29,30}$, current pregnancy, 28,33 pain during testing, ${ }^{29}$ vascular disease, $, 32,37$ use of certain medicaitons, ${ }^{32,37,}$ ${ }^{38}$ neuromuscular disease, ${ }^{33,}, 34$ diabetes, ${ }^{34}$ conditions known to effect balance, ${ }^{34-35,}$ currently assisted ambulation, ${ }^{37}$ positive ligamentous testing, ${ }^{37}$ grade III ankle sprain, ${ }^{37}$ other treatment for ankle injury, $, 38,40$ and history of contralateral ankle sprain. ${ }^{40}$

Studies ranged from 1 to 8 on the PEDro scale with an average score of 5.81. A summary of scores can be found in Table D5. All individual items can be found in Tables D6-21. When evaluating the studies there were some discrepancies between the two raters, especially among items 6-8. Five studies evaluated the use of Maitland mobilization. ${ }^{30,32} 34$, 35, 41 Six studies evaluated Mulligan's mobilization with movement (MWM). ${ }^{26,29,31,37,39,40}$

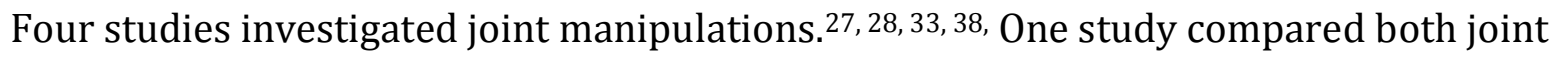
manipulations and mobilizations. ${ }^{36}$ Eight studies also focused on CAI. ${ }^{28,31,33-36,38, ~} 40$ Effect sizes for 11 of the 16 studies included were calculated. ${ }^{27-31,34-38,40}$ Only 4 of those studies showed a benefit of treatment as the $95 \%$ CI for effect sizes did not cross $0.36-38,40$ All effect sizes can be found in Tables D22-32. 
Of these studies, 13 subjects were randomly allocated into treatment groups. 26 _30,3234, 36, 38-41 Five of these studies concealed group allocation. ${ }^{27,28,30,32,34}$ Thirteen studies had similar baseline scores for subjects. $26-30,32,33,35-37,39-41$ Only two studies were able to successfully blind subjects so that they were unable to determine which treatment group. ${ }^{29}$, ${ }^{36}$ No groups were able to blind the therapists as they needed to perform the mobilization. Twelve of the studies blinded the assessors during evaluation. A follow up rate of at least $85 \%$ was present in a majority of the studies. $26-30,32,35,36,38-41$

Joint Mobilization

There were five studies that evaluated the effect of regular joint mobilization. Cosby et al., ${ }^{30}$ Green et al.,32 and Yeo et al. ${ }^{41}$ evaluated a combined total of 68 subjects with acute ankle sprain. Cosby et al. ${ }^{30}$ divided 17 subjects into a control and talocrural joint mobilization group. Each subject had a previous lateral ankle sprain and was immobilized with a walking boot. The control group had no treatment and the joint mobilization group received only a single session of mobilization. This study evaluated dorsiflexion ROM and posterior talar translation and found no significant differences between groups. The PEDro scale rating for this study was $8 / 10$ which indicated good methodological quality (Table D10). None showed a true effect of the treatment when compared between groups and within groups (Table D25).

Green et al. ${ }^{32}$ randomized 38 subjects into a control group, who received RICE treatment, and an anterioposterior mobilization group. The mobilization group received 6 treatments over a 2 week period. Anteroposterior joint mobilizations improved ankle dorsiflexion ROM when compared to a control group. There was not enough information 
presented in the article to calculate effect size. The PEDro scale rating is $7 / 10$, which indicated fairly good methodological quality (Table D12).

Yeo et al. ${ }^{41}$ randomized the order in which 13 subjects with unilateral sub-acute ankle sprain received a joint mobilization treatment, a manual contact treatment, a no contact control treatment. Each treatment was given once to each subject with a 48 washout period between treatments. There was a significant increase of WB dorsiflexion ROM of about $9.6 \mathrm{~mm}$. Pressure pain threshold showed an increase of $17.76 \%$ that was statistically significant. A slight decrease in VAS score was noted but the decrease was not statistically significant. There was not enough information reported in the study to determine effect size. The PEDro rating for this article is $5 / 10$ which indicated fair methodological quality (Table D21).

Both Hoch et al. ${ }^{34,35}$ examined joint mobilizations in subjects with chronic ankle instability. In the 2011 study Hoch et al. ${ }^{34}$ used a crossover design with 20 subjects. Each subject received both a grade III joint mobilization treatment and a rest treatment (control). A significant increase in dorsiflexion ROM in the patients undergoing joint mobilization was noted. Posterior glide showed no significant change. The PEDro scale rating for this article was 5/10 which indicates fair methodological quality (Table D14). The effect sizes reported crossed zero and did thus did not result from the treatment given (Table D27).

Hoch et al. ${ }^{35}$ examined 12 subjects who all received 2 types of joint mobilization, a grade II traction technique and a grade III posterior mobilization, for 6 treatments over a period of 8 to 20 days. Dorsiflexion ROM (WB) was assessed and no significant values were found. The effect sizes reported crossed zero and did thus did not result from the treatment 
given (Table D15). The PEDro Scale rating for this article was $5 / 10$ which shows a fair amount of methodological study (Table D28).

Overall these studies had a PEDro scale range of 5-8, with an average of $6.30,32,34,35$, ${ }^{41}$ All effect sizes that could be calculated were small and encompassed $0.30,34,35,41$ Four of these studies found an increase in dorsiflexion ROM. ${ }^{32,34,35,41}$ Only Yeo et al. ${ }^{41}$ examined pain levels and found a significant improvement. Two studies evaluated posterior glide but a significant difference was not found..$^{30,34}$

Mulligan's Mobilization with Movement

There were six studies that evaluated mobilizations with movement. ${ }^{26,} 29,31,37,39,40$ Akre et al., ${ }^{26}$ Collins et al., ${ }^{29}$ Nambi et al., ${ }^{37}$ and Reid et al. ${ }^{39}$ performed MWM on subjects with acute lateral ankle sprains. Akre et al. ${ }^{26}$ randomized 30 subjects with unilateral ankle sprains into a mobilizations with movement in weight bearing (MWM-WB) group and a mobilizations with movement in non-weight bearing (MWM-NWB) group. A posteroanterior glide to the tibia over 10 consecutive days was used. Both treatments reduced pain levels and increased ROM. The increase in plantarflexion ROM was found to be significant while the increase in dorsiflexion ROM was not statistically significant. The treatments also found a decrease in pain. While the differences between groups were not statistically significant, MWM-WB typically was found to have a slightly better benefit than MWM-NWB. ${ }^{19-20,29,42}$ The effect sizes could not be calculated for this study. The PEDro score this study as a 6/10 (Table D6). This shows that the study has a fair methodological quality.

Collins et al. ${ }^{29}$ used 16 subjects with a grade II lateral ankle sprain who received a MWM-WB treatment, placebo treatment, and no treatment. Each treatment was only 
performed one time. Dorsiflexion ROM was found to have a significant increase in the MWM-WB treatment group. Pain was found to have a significant improvement in the placebo group. These findings suggest that pain relief does not occur with this type of treatment. The effect sizes reported crossed zero and thus did not show a true effect of treatment (Table D24). The PEDro scale rating on this study was $7 / 10$, which shows good methodological quality (Table D9).

Nambi et al. ${ }^{37}$ randomly assigned 30 field hockey players with acute lateral ankle sprains to a kinesiotape group and a MWM-WB group. Treatments were given over the course of one week. Pain rating had significantly decreased by $75.9 \%$ in the MWM-WB group compared to a 55.69\% reduction in the kinesiotape group. Dorsiflexion improved by $71.07 \%$ with MWM-WB compared to a $27.64 \%$ increase with kinesiotape. Effect sizes showed that both Kinesiotape and MWM-WB did cause the benefits found in the study (Table D30). In both pain rating and dorsiflexion ROM MWM-WB showed a larger effect over kinesiotape. The PEDro score was 3/10, which shows poor methodological quality (Table D17).

Reid et al..$^{39}$ treated 23 subjects with unilateral ankle sprains each with MWM-WB treatment and sham joint mobilizations for 1 treatment. A 1 week washout period was used to prevent inter-treatment bias. A significant change was found in dorsiflexion range of motion in the MWM-WB group. The effect sizes were unable to be calculated. The study has a PEDro rating of 7/10, which shows a fair methodological quality (Table D19).

Gilbreath et al., ${ }^{31}$ and Vicenzino et al. ${ }^{40}$ performed mobilizations with movement on subjects with chronic ankle instability. Gilbreath et al. ${ }^{31}$ treated 11 subjects who reported CAI and were physically active. They received 3 treatments over a 2 week period with no 
significant difference in dorsiflexion ROM. The effect size calculated were small and encompassed 0 (Table D26). The PEDro scale rating for this study was a $1 / 10$, which shows poor methodological quality (Table D11).

Vicenzino et al. ${ }^{40}$ performed MWM-WB treatment, MWM-NWB treatment and no treatment control on 16 subjects with a history of recurrent lateral ankle sprain. Between each treatment a 48 hour washout period was conducted. Dorsiflexion (WB) was reduced by $26 \%$ of the unaffected side deficit. Posterior talar glide significantly reduced in MWM treatments. This deficit was reduced by about $50 \%$ in the NWB group compared to $55 \%$ in the WB group. The PEDro scale rating is a $6 / 10$, which shows fair methodological quality (Table 20). The effect sizes that did not encompassed 0 showed a moderate effect on posterior glide in MWM-WB and a strong effect on posterior glide in MWM-NWB (Table 32).

Overall the PEDro scores for the MWM group ranged from 1 to 7 and had an average score of $5 .{ }^{26,29,31,37,39,40}$ In 4 of these studies effect sizes were calculated. ${ }^{29,31,37,40}$ However only Nambi et al. ${ }^{37}$ and Vicenzino et al. ${ }^{40}$ found effect sizes with CI that did not cross 0 . Five of these studies found a significant increase in dorsiflexion ROM. 29, 31, 37, 39, 40 Only Akre et al. ${ }^{29}$ found non-significant increases for dorsiflexion ROM and significant increases for plantarflexion ROM. Pain improved in $3^{26,29,37}$ studies but only 2 were statistically significant. ${ }^{26,37}$ Posterior glide also showed improvements in Vicenzino et al.40

Joint Manipulations

Four studies examined the benefits of joint manipulations on lateral ankle sprains. ${ }^{27,}$ ${ }^{28,33,38}$ Andersen et al. ${ }^{27}$ randomized 52 subjects with acute lateral ankle sprain into a high velocity, low amplitude (HVLA) manipulation group and a no treatment group. Only one 
treatment was provided. Dorsiflexion ROM and ankle torque reported did not change. The effect sizes indicated that the treatment had no effect on the outcomes. (Table D22) The PEDro rating was 8/10, which shows a strong methodological quality (Table D7).

Beazell et al. ${ }^{28}$ examined 43 subjects that were placed into a proximal tibiofibular manipulation group (PTFM), a distal tibiofibular manipulation group (DTFM), and a no treatment group. Each group received 3 treatments over the course of one week. There was no significant difference between groups in dorsiflexion ROM but a significant increase was found when comparing the groups over time. The effect sizes indicated that no treatment had an effect on outcomes. (Table D23) The PEDro rating is 8/10, which shows a strong methodological quality (Table D8).

Grindstaff et al. ${ }^{33}$ examined 43 subjects with CAI that were randomized into a proximal tibiofibular joint manipulation group, distal joint manipulation group, or no treatment. Only 1 treatment was given and found that there was a significant difference in $\mathrm{H} / \mathrm{M}$ ratios in the soleus after intervention. This study did not provide enough information to calculate effect sizes. The PEDro rating is $4 / 10$ and shows poor methodological quality (Table D13).

Pellow et al. ${ }^{38}$ examined 30 subjects with both acute and chronic ankle sprains. This study compared an ankle mortise separation group to a control group. Each group received 8 treatments over a 4 week period. An increase in dorsiflexion ROM and decrease in pain was found to be significant in the 1 month follow up for the adjustment group, however both groups improved over time. The effect sizes were large for improvement in pain rating scales, dorsiflexion ROM improvements, and improvements with pain pressure threshold (algometer)(Table D30). The adjustment group showed a large effect with the 
McGill pain questionnaire, and dorsiflexion ROM immediately after treatment. At 1 month follow up, only dorsiflexion ROM and pain pressure threshold continued to have a strong effect. The PEDro rating is a $6 / 10$, which indicated fair methodological quality (Table D18).

Overall, the joint manipulation group had PEDro scores that ranged from 4 to 8, with an average of 6.5.27, 28, 33, 38 Effect sizes could be calculated for three of these studies ${ }^{27}$, but only Pellow et al. ${ }^{38}$ had effect sizes that did not encompass 0 . A significant increase in dorsiflexion ROM was found in Pellow et al..$^{38}$ Grindstaff et al. ${ }^{33}$ determined that $\mathrm{H} / \mathrm{M}$ ratios were significant for the soleus muscle but not for the fibularis longus. This could indicate that after joint mobilizations the soleus is active and may assist in increasing ROM. Significant pain and dorsiflexion was found in Pellow et al. ${ }^{38}$ Grindstaff et al. ${ }^{33}$ showed improvements for $\mathrm{H} / \mathrm{M}$ ratio for the soleus but not for the fibularis longus. No significant improvements were found for dorsiflexion in the remaining studies. ${ }^{27,} 28$ Joint Mobilization versus Joint Manipulation

Finally Marron-Gomez et al. ${ }^{36}$ examined both high velocity, low amplitude (HVLA) manipulation group and joint mobilization group compared to a placebo group. They used 52 subjects with chronic ankle instability for this study. Both MWM-WB and HVLA groups had a significant increase in dorsiflexion ROM when compared to the control. The MWMWB showed a moderate effect size while the HVLA group showed a small effect size. This means that MWM-WB would likely have larger effect on the population. All effect sizes evaluated can be found in Table D29 The PEDro rating for this study is $7 / 10$, which shows good methodological quality (Table D16).

Joint mobilizations and manipulations cause an increase in dorsiflexion range of motion, an improvement in pain measures and a decrease in posterior talar glides. The 
studies evaluated reported mixed results about the effectiveness of joint mobilizations on improving these outcomes. Overall joint mobilizations provided a quicker immediate effect but are often most effective when multiple treatment sessions were used over time. Use of manipulations provided smaller improvements and were usually found at a minimum of 24 hours post treatment. The studies had an average age of 23.95. Only two studies evaluated an active or athletic population. ${ }^{33,37}$ Overall studies showed a wide variety of PEDro scores and only 4 effect sizes that did not encompass 0 .

\section{DISCUSSION}

The purpose of this study was to examine and analyze the current literature on joint mobilizations and manipulation techniques on treatment of lateral ankle sprains, both acute and chronic. Specifically their effect on mechanical instability, the effectiveness therein, and whether or not type of mobilization show a treatment in various outcomes.

Three hypotheses were used in this study. The first was that the use of joint mobilization would cause a change in the lateral ankle sprain. The second was that the mobilization type used for treatment will show a difference in outcome. The last hypothesis was that there would be enough information in each study to use the PEDro scale and calculate effect size. The first was confirmed. The second hypothesis needs more comparative studies to be properly confirmed. The last hypothesis was unconfirmed as effect sizes could only be calculated for 11 studies.

Of the studies evaluated most found significant improvements in at least one of the following: dorsiflexion ROM, posterior glide, pain levels, and H/M ratios. The 11 studies where effect sizes could be calculated have shown in general that these benefits of joint mobilization techniques do not show a true effect from treatment and thus cannot be 
recommended for the clinician's use. Joint mobilization studies showed confidence intervals that encompassed zero and low methodological quality. MWM studies showed a wide range of methodological quality and two studies showed large effect sizes. These studies do have more evidence than joint mobilization however a recommendation cannot be made for clinician use. Joint manipulation studies showed moderate to high methodological quality but only one study found large significant effect sizes that did not encompass zero. Manipulations cannot be recommended for clinican use with the evidence found in this study. Joint manipulation vs MWM showed large effect sizes but moderate methodological quality. It is because of these findings that there is not a strong indication for joint mobilization or manipulation use in the lateral ankle sprain despite the significant benefits that were found in each study. In general, when a study was found to have a high methodological quality the effect sizes were small and/or encompassed 0 . If the study had large effect size that did not encompass 0 , they often had small or moderate methodological quality.

PEDro scale scores ranged from 1 to 8 and had an average of 5.81 among all the studies, indicating that the overall methodological quality of the studies was moderately poor. One of the common flaws noted in methodology was lack of subject and therapist blinding. Effectiveness of these treatments was evaluated by various measures which further confounds this study, despite the fact that only outcomes that pertained to mechanical instability were evaluated. These included dorsiflexion ROM, with one study evaluating plantarflexion ROM, pain measures, posterior talar displacement or glide, torque, and $\mathrm{H} / \mathrm{M}$ ratios. 
Most of the studies had positive outcomes however some of the reported findings were not statistically significant or showed no change. There were 11 studies that found a benefit for dorsiflexion range of motion ${ }^{26,29,32,34-41}$ but 4 did not find these outcomes statistically significant or there was no change to ROM.27, 28, 30, 31 Pain measures showed improvements in 5 studies $26,29,37,38,41$ Posterior talar displacement and stiffness improved in 2 studies. ${ }^{38,40}$ Two others found it to be not statistically significant. ${ }^{30,34}$ Torque was found to not have any change when evaluated in Andersen et al. ${ }^{27} \mathrm{H} / \mathrm{M}$ ratio showed improvement in recruitment from fibularis longus and soleus but was not statistically significant. Effect sizes were able to be evaluated on 11 studies and demonstrated mixed results with 95\% CI. All effect sizes can be found in tables D22-D32.

Joint Mobilizations

Four of five studies that examined joint mobilizations found a significant increase in dorsiflexion ROM. ${ }^{32,34,35,41}$ The increase of dorsiflexion ROM can be caused by improvement in arthrokinematics, removal of adhesions, and stretching of damaged tissues to allow for more joint movement. $8,13,14,15$ An increase in dorsiflexion range of motion will help the patient return quicker to normal function and joint movement. ${ }^{8,12-13}$ Decreased dorsiflexion range of motion has also been shown to be a risk factor in recurrent ankle sprains ${ }^{8,43}$ Thus, returning the joint back to normal motion is important for the recovery and prevention of re-injury. Pain measures significantly decreased in the study done by Yeo et al. ${ }^{41}$ Pain reduction may be due to an increase in synovial fluid production which allows the joint to move properly. A reduction in pain levels can often increase ROM and function as the patient is more comfortable performing those tasks. Posterior glides found no significant difference in the studies evaluated. 
Of the 3 studies ${ }^{30,34,35}$ where both effect sizes and PEDro scores could be calculated, all had small effect sizes that encompassed zero. In addition these studies had an average PEDro score of 6, with a range of 5-8. The benefits found for these studies may be due to other factors and not solely the effect of the joint mobilization. Due to this no recommendations for the clinician can be made.

There are some factors that could cause the low effect sizes for these studies. The first is performing a single treatment session or multiple sessions. Typically with any manual therapy technique multiple treatments are needed for improvements to be shown. This is especially the case in the use of joint mobilizations. Of these studies only Hoch et al ${ }^{35}$ preformed multiple treatments. Another factor that could affect outcomes is the grade of mobilization used. All studies used a Maitland grade III mobilization for treatment which is what is typically used in the clinical setting. Only Hoch et $\mathrm{al}^{35}$ used the addition of a grade II traction mobilization. Joint mobilizations are also never used as a standalone treatment for treating the ankle only Cosby et al..$^{30}$ allowed subjects to receive standard RICE, strengthening, and proprioceptive training program. However this was optional and not all subjects completed participated. Patient populations could also have been a factor as to why effect sizes were low and crossed 0 . Cosby et al. ${ }^{30}$ examined acute lateral ankle sprains with either grade I or II ankle sprains that were randomly assigned to groups. The lack of changes between groups could be attributed to the fact that the control or experimental may have had more grade I ankle sprains over grade II ankle sprains. Both Hoch et al $\operatorname{articles}^{34,35}$ examined subjects with chronic ankle instability. The number of patients might have also weakened effect sizes. The average subjects between these three studies was only 
16.3. Blinding of assessors was not done in Hoch et al. ${ }^{35}$ This could have caused an examiner bias causing the significant improvements noted.

Mobilizations with Movement

This joint mobilization technique can be performed both in weight bearing and nonweight bearing. In most of the literature and in clinical practice MWM is most commonly performed in weight-bearing. Five of the studies examined showed a significant increase in dorsiflexion ROM. $29,37,39,40$ Akre et al. ${ }^{26}$ found a non-significant improvement in dorsiflexion ROM. As mentioned earlier normal dorsiflexion ROM can benefit the arthrokinematics, joint adhesions, and function in the ankle joint. This can benefit the patient by improving mechanics thus improving function and reduce injury. Significant improvements in pain were found in Akre et al. ${ }^{26}$ and Nambi et al. ${ }^{37}$ Vicenzino et al. ${ }^{40}$ also found improvements in posterior glide. Of these studies only 2 compared MWM-WB and MWM-NWM ${ }^{26,40}$ while the remaining studies only evaluated MWM-WB. ${ }^{29,31,37,39}$ Both Vicenzino et al..$^{40}$ and Akre et al. ${ }^{26}$ found that both MWM treatments were beneficial with no significant differences between groups but slight improvements were noted from MWMWB over MWM-NWB. Only 3 studies ${ }^{26,31,37}$ used multiple treatments and 2 showed statistically significant benefits. ${ }^{26,37}$ Both dorsiflexion and pain improved with the use of multiple treatments of MWM. However, there have been some studies that have found slight improvements but no significant differences. ${ }^{29,31}$

In 4 of these studies both effect sizes and PEDro scores were able to be calculated. ${ }^{29.31 .37,40}$ PEDro scores showed a range of 1 to 7 with an average of 4.25. Two of these studies had small effect sizes that encompassed zero. ${ }^{29,31}$ Nambi et al. ${ }^{37}$ showed a large effect in both within group dorsiflexion ROM and pain levels and between group 
dorsiflexion ROM. These effect sizes favored MWM-WB over the kinesiotape treatment. However, the methodological quality for this study is very low. The study could have a variety of biases that were not protected against. Due to this these effect sizes cannot be used to recommend this treatment to the athletic trainer. Vicenzino et al. ${ }^{40}$ had effect sizes that did not encompass 0 in only two within group measures: posterior glide MWM-WB and MWM-NWB. MWM-NWB showed a slightly larger effect. However when compared to controls the effect sizes encompassed 0 . Methodological quality was moderate. These studies do not have enough evidence to support a strong recommendation for the therapist.

The lack of supporting evidence found can be due to a variety of factors. Only 2 of these studies had multiple treatment sessions. ${ }^{31,37}$ Significant benefits cannot be expected to be found after only one joint mobilization treatment this could be a reason as to why effect sizes are small or cross 0 . Only Nambi et al. ${ }^{37}$ gave all patients a standard treatment of ultrasound. However neither ultrasound, nor joint mobilizations are used alone for the treatment of ankle sprains. The patient population for these studies were fairly small with an average of 18.25 . This could limit the results found as the populations may not have been heterogeneous. The grade of ankle sprains can also have an effect on the amount of benefit found. These studies had a wide variety in the severity of ankle sprains. Grade II ankle sprains, previous history of ankle sprain, $\mathrm{CAI}$, and an ankle sprain from 10 days to 7 weeks after injury were included in these patient populations. The variety may have diffused some of the findings. Both Gilbreath et al. ${ }^{31}$ and Nambi et al. ${ }^{37}$ did not blind the assessors. This may be one of the reasons why Nambi et al. ${ }^{37}$ found such large benefits. 
Joint Manipulation

The studies evaluated most found a significant increase in range of motion, reduction in pain, and significant $\mathrm{H} / \mathrm{M}$ ratios. A significant increase in dorsiflexion ROM was found in Pellow et al. ${ }^{38}$ Grindstaff et al. ${ }^{33}$ determined that $\mathrm{H} / \mathrm{M}$ ratios were significant for the soleus muscle but not for the fibularis longus. This could indicate that after joint mobilizations the soleus is active and may assist in increasing ROM in plantarflexion. It can also indicate a relaxation of the fibularis longus muscle. Due to the function and placement of this muscle a decreased firing pattern could lead to functional instabilities and a decreased ability for the ankle to prevent ankle inversion. Significant pain reduction was also found in Pellow et al. ${ }^{38}$

Effect sizes and PEDro scores could be found for only 3 of these studies. $27,28,38 \mathrm{In}$ two of the cases the effect sizes were small and encompassed zero. ${ }^{27,28}$ These two studies also had good methodological quality. In Pellow et al. ${ }^{38}$ Large effect sizes were found for dorsiflexion and pain within groups and between groups. Effect sizes favored the adjustment group. This study had a moderate methodological quality. As noted earlier due to these findings no recommendations can be made for the clinician.

The lack of findings can be attributed to many factors. Only Pellow et al. ${ }^{38}$ performed multiple treatments and also was the only study to have effect sizes that showed a treatment effect. None of these studies stated that other treatments were done in addition to the joint mobilization technique. However in Pellow et al. ${ }^{38}$ the control group received an ultrasound treatment. In 2 of these studies a HVLA manipulation technique was used. ${ }^{27,28}$ Pellow et al. ${ }^{38}$ examined an adjustment technique. Since two types of manipulation techniques were evaluated generalizations about the adjustment technique cannot be 
made as only one study evaluated this technique. The studies had an average patient group of 41.6. This mobilization group had the largest patient group. This helps with the significance of the studies as it is likely that these populations are more heterogeneous. Ankle grades were not specified for these studies. At least 2 of the studies had inclusion criteria that stated previous ankle sprain or chronic instability was used. In Pellow et al.,38 they looked at both acute and chronic ankle sprains. The more heterogeneous the patient populations, typically the easier it is to generalize the outcomes for clinical use. However, this can cause a dilution of outcome benefits.

Joint Mobilization versus Manipulation

Both MWM-WB and HVLA groups showed a significant increase in dorsiflexion ROM when compared to the placebo but no significant differences were found when compared to each other. In this study, the HVLA treatment had a large effect size when compared to the placebo treatment, where MWM showed a moderate effect. In both cases the effect size encompassed 0 . The PEDro scale rating on this study is $7 / 10$ which indicates good quality. This is the only study that directly compared a joint mobilization technique (MWM) and a joint manipulation (HLVA) technique. Again in this study only 1 treatment was done and no rehabilitation program was mentioned. Typically in the treatment of an injury mobilizations are done over multiple treatment sessions and with the addition of a strengthening and proprioception rehabilitation program. The population in this study was fairly large and they only evaluated patients with CAI. This study indicated that the use of HVLA manipulations were beneficial and had a good quality study. However when these results are coupled with the results in the manipulation group a recommendation cannot be made for treatment use. 
Joint Mobilization Effect on Tissues

The effect on joint mobilizations on tissues has not been fully evaluated. Both Hoch et al studies ${ }^{34,35}$ theorized that joint mobilizations changed joint arthrokinematics when dorsiflexion was increased. Joint mobilizations often work the non-contractile tissues surrounding the area to help increase dorsiflexion ROM and posterior glides. This is believed to be caused by a plastic deformation and an increase in extensibility and flexibility to the tissues. ${ }^{32}$ They also believe an increase in functional aspects could be due an increase in the stimulation of articular mechanoreceptors afferent pathways. MWM is also thought to help to improve talar position especially with improvements to the gastrocsoleus complex. ${ }^{31}$ Improvements to the gastroc-soleus complex is especially noted in MWM technique due to active dorsiflexion especially in the weight bearing technique. With joint manipulations some of the structures are thought to be snapped back into their proper position in addition to trying to increase plastic deformation and tissue extensibility. ${ }^{44}$ These changes alter the arthrokinematics of the joint due to the change in structure. Limitations of the Study

There were a total of 16 studies that fit the criteria for inclusion. Overall methodological quality was found to be poor. This is likely due to the difficulty in blinding both the therapist and subject. For most of the studies evaluated a "no" was recorded for criteria 3, 5, and 6 on the PEDro Scale. Criteria 3 refers to allocation being concealed. Often this was not stated and was rated as a "no". Criteria 5 and 6 were blinding of subjects and blinding of therapists. It is impossible for the therapist to be blinded as they need to know which treatment to apply to the patient. The patient can be semi-blinded but would likely be able to determine which group they were in unless a sham or placebo group were also 
added. Even in these cases, some studies reported that subjects were able to determine which treatment group they were in by the end of the study. PEDro ratings may also have contributed to poor methodological outcomes. The use of two raters who underwent a PEDro scale training program was used to try to prevent bias. However the two raters still had discrepancies over some PEDro scores initially. All differences were discussed until an greement was reached by the two raters.

Another limitation of this study was a lack of effect sizes that did not cross 0 . Effect sizes for 11 of the 16 studies found were able to be calculated. However many of these did not determine that the treatment was the direct cause of improvements because they crossed zero. This could be due to the poor methodological quality or poor treatment outcomes. Of the 5 studies the effect sizes could not be calculated because mean and standard deviation values could not be found within the study.

In the systematic search, only 7 databases were used and only studies found in English were included into the study. In other databases more studies may have been found to include. Studies written in other languages could also have applied to this study but had to be excluded as the investigator did not know a second language.

\section{Clinical Relevance}

The studies evaluated determined that joint mobilizations and manipulations generally show an increase in dorsiflexion ROM, posterior glide, pain relief, and H/M ratios. However due to poor methodological quality and most effect sizes encompassing 0 no recommendations can be made for the clinician. Only 7 studies had multiple treatment sessions and only 1 truly evaluated the use of a standard treatment in addition to joint mobilizations. In typical clinic use, joint mobilizations are done over multiple treatment 
sessions and with the addition of a comprehensive rehabilitation program. More studies should be done with multiple treatment sessions and the use of a standard rehabilitation program. This would show if the standard clinical use of joint mobilizations shows a true benefit.

Regardless of effect sizes, most studies did show a statistically significant benefit in at least one mechanical outcome measured. The evidence found in this report has the most support for MWM-WB. Normal joint mobilization also has a fair amount of evidence supporting its use. The use of joint manipulations has the least amount of evidence to support a benefit. Previous reviews ${ }^{9,19,20}$ have supported the use of joint mobilizations and MWM. The use of joint mobilizations and manipulations has shown good evidence in the past to support its use. Clinically the addition of joint mobilizations to a standard treatment protocol for the lateral ankle sprain has previously shown benefits over time.

\section{CONCLUSION}

Athletic trainers commonly use manual therapy techniques to assist athletes who are recovering from injury. Many manual therapy techniques can be used to benefit healing and improve patient function. It is important for the athletic trainer or clinician to understand how effective and the potential benefits of using joint mobilizations in the lateral ankle sprain. Joint mobilizations increase dorsiflexion range of motion, decrease pain, increase posterior glide, and increase $\mathrm{H} / \mathrm{M}$ ratios. However most of these studies had results that were not statistically significant or effect sizes showed that the treatment did not necessarily cause the benefit noted in the study. Research with a higher methodological quality would help to determine if the outcomes found in joint mobilizations are caused due to the treatment or due to other errors. 


\section{REFERENCES}

1. Waterman BR, Owens BD, Davey S, Zacchilli, Belmont PJ. The epidemiology of Ankle Sprains in the United States. J Bone Joint Surg Am. 2010;92:2279-84.

2. Cameron KL, Owens BD, DeBerardino TM. Incidence of ankle sprains among activeduty members of the united states armed services from 1998 through 2006. J Athl Train. 2010;45(1):29-38.

3. Ekstrand J, Hägglund M, Waldén M. Injury incidence and injury patterns in professional football: the UEFA injury study. Br J Sports Med. 2011;45:553-558.

4. Cloke DJ, Ansell P, Avery P, Deehan D. Ankle injuries in football academies: a threecentre prospective study. Br J Sports Med. 2011;45:702-708.

5. Beynnon BD, Vacek PM, Murphy D, Alosa D, Paller D. First-time inversion ankle ligament trauma: the effects of sex, level of competition, and sport on the incidence of injury. Amer J Sports Med. 2005;33(10):1485-1491.

6. Rumpf MC, Cronin J. Injury incidence, body site, and severity in soccer players aged 6-18 years: implications for injury prevention. Strength Cond J. 2012;34:20-31.

7. Tanaka H, Mason L. Chronic Ankle Instability. Orthop Trauma. 2011;25(4):269-278.

8. Hertel J. Functional anatomy, pathomechanics, and pathophysiology of lateral ankle instability. J Athl Train. 2002;37(4):364-375.

9. Martin RL, Davenport TE, Seth SP, Wukich DK, Godges JJ. Ankle Stability and Movement Coordination Impairments: Ankle Ligament Sprains. J Orthop Sports Phys Ther. 2013;43(9):A1-A4Q.

10. Maffulli N, Longo UG, Petrillo S, Denaro V. Lateral ankle instability. Ortho Trauma. 2012;26(1):20-24.

11. Kaminski TW, Hertel J, Amendola N, Docherty CL, Dolan MG, Hopkins T, Nussbaum E, Poppy W, Richie D. National Athletic Trainers' Association Position Statement: Conservative management and prevention of ankle sprains in athletes. J Athl Train. 2013;48(4):528-545.

12. Hubbard TJ, Cordova M. Mechanical instability after an acute lateral ankle sprain. Arch Phys Med Rehabil. 2009;90;1142-1146. 
13. Hubbard TJ, Hertel J. Mechanical contributions to chronic lateral ankle instability. Sports Med. 2006;36(3):263-277.

14. Denegar CR, Hertel J, Fonseca J. The effect of lateral ankle sprain on dorsiflexion range of motion, posterior talar glide, and joint laxity. J Orthop Sports Phys Ther. 2002;32(4):166-173.

15. Brown C, Padua D, Marshall SW, Guzkiewicz K. Individuals with mechanical ankle instability exhibit different motion patterns than those with functional ankle instability and ankle sprain copers. Clin Biomech. 2008;23:822-831.

16. Hultman K, Faltstrom A, Oberg U. The effect of early physiotherapy after an acute ankle sprain. Advances Physiothe. 2010;12:65-73.

17. Cleland JA, Mintken P, Mcdevitt A, Bieniek M, Carpenter K, Kulp K, Whitman JM. Manual physical therapy and exercise versus supervised home exercise in the management of patients with inversion ankle sprain: a multicenter randomized clinical trial. J Orthop Sports Phys Ther. 2013;43(7): 443-455.

18. Delahunt E, Cusack K, Wilson L, Doherty C. Joint mobilization acutely improves landing kinematics in chronic ankle instability. J Amer College Sports Med. 2013:514519.

19. Hing W, Bigelow R, Bremner T. Mulligan's mobilisation with movement: a review of the tenets and prescription of MWMs. NZ J Physioth. 2008;36(3):144-164.

20. Hoch MC, McKeon PO. The effectiveness of mobilization with movement at improving dorsiflexion after ankle sprain. J Sport Rehabili. 2010;19:226-232.

21. Maher CG, Sherrington C, Herbert RD, Moseley AM, and Elkins M. Reliability of the PEDro scale for rating quality of randomized controlled trials. Phys Ther. 2003;83:713-721.

22. PEDro Scale. 1999 Available at: www.pedro.org.au/english/download/pedro-scale. Accessed November, 29, 2013.

23. Elkins MR, Moseley AM, Sherrington C, Herbert RD, Maher CG. Growth in the physiotherapy evidence database (PEDro) and use of the PEDro scale. Br J Sports Med. 2013;47(4):188-189. 
24. De Morton NA. The PEDro scale is a valid measure of the methodological quality of clinical trials: a demographic study. Austr J Physiother. 2009; 55: 129-133.

25. Witte RS, Witte JS. Statistics. Eighth Edition. Danvers, MA: John Wiley \& Sons; 2005.

26. Akre AA, Jeba C, and Khatri SM. Comparative effectiveness of mulligan's mobilization in weight bearing and non-weight bearing in the treatment of ankle sprains-a randomized clinical trial. Ind J Physiother Occupational Ther. 2008;2(4):1-4.

27. Andersen S, Fryer GA, McLaughlin P. The effect of Talo-Crural joint manipulation on range of motion at the ankle joint in subjects with a history of ankle injury. ACO. 2003;11(2):57-62.

28. Beazell JR, Grindstaff TL, Sauer LD, Magrum E, Ingersoll CD, and Hertel J. Effects of a proximal or distal tibiofibular joint manipulation on ankle range motion and functional outcomes in individuals with chronic ankle instability. J Orthop Sports Phys Ther. 2012;42(2):25-134.

29. Collins N, Teys P, Vicenzino B. The initial effects of a Mulligan's mobilization with movement technique on dorsiflexion and pain in subacute ankle sprains. Man Ther. 2004;9:77-82.

30. Cosby NL, Koroch M, Grindstaff TL, Parente W, Hertel J. Immediate effects of anterior to posteror talocrural joint mobilizations following acute lateral ankle sprain. J Man Manip Ther. 2011;19(2):76-83.

31. Gilbreath JP, Gaven SL, Van Lunen BL, Hoch MC. The effects of mobilization with movement on dorsiflexion range of motion, dynamic balance, and self-reported function in individuals with chronic ankle instability. Man Ther. 2013; 1-6.

32. Green T, Refshauge K, Crosbie J, Adams R. A randomized controlled trial of a passive accessory joint mobilization on acute ankle inversion sprains. Phys Ther. 2001;81:984-994.

33. Grindstaff TL, Beazell JR, Sauer LD, Magrum EM, Ingersoll CD, Hertel J. Immediate effects of a tibiofibular joint manipulation on lower extremity H-reflex measurements in individuals with chronic ankle instability. J Electromyogr Kinesiol. 2011;21:652-658.

34. Hoch MC, McKeon PO. Joint mobilization improves spatiotemporal postural control and range of motion in those with chronic ankle instability. J Orthop Res. 2011;29(3):326-332. 
35. Hoch MC, Andreatta RD, Mullineaux DR, English RA, McKeon JMM, Mattacola CG, and McKeon PO. Two-week joint mobilization intervention improves self-reported function, range of motion and dynamic balance in those with chronic ankle instability. J Orthop Res. 2012;30(1):1798-1804.

36. Marrón-Gómez D, Rodríguez-Femández AL, Martin-Urrialde JA. The effect of two mobilization techniques on dorsiflextion in people with chronic ankle instability. Phys Ther Sport.2014;1-26.

37. Nambi GS, Shah BT. Kinesio taping versus Mulligan's mobilization with movement in sub-acute lateral ankle sprain in secondary school hockey players-comparative study. Inter J Pharmaceutical Sci Health Care. 2012;2(2):136-149.

38. Pellow JE, Brantingham JW. The efficacy of adjusting the ankle in the treatment of subacute and chronic grade I and grade II ankle inversion sprains.J Mani Physiolog Therap. 2001;24(1):17-24.

39. Reid A, Birmingham TB, Alcock G. Efficacy of mobilization with movement for patients with limited dorsiflexion after ankle sprain: a crossover trail. Physiother Can. 2007;59:166-172.

40. Vicenzino B, Branjerdporn M, Teys P, Jordan K. Initial changes in posterior talar glide and dorsiflexion of the ankle after mobilization with movement in individuals with recurrent ankle sprains. J Orthop Sports Phys Ther. 2006;36(7):464-471.

41. Yeo HK, Hypoalgesic effect of a passive accessory mobilization technique in patients with lateral ankle pain. Man Ther. 2011;16:373-377.

42. Baker RT, Nasypany A, Seegmiller JG. The mulligan concept: mobilization with movement. International J Athl Ther Train. 2013;January:50-54.

43. Brinkman RE, Evans TA. History of ankle sprain as a risk factor of future lateral ankle sprain in athletes. J Sport Rehabil. 2011;20:384-388.

44. Fryer GA. Mudge JM, McLaughlin PA. The effect of talocrural joint manipulation on range of motion at the ankle. J Manipulative Physiol Ther. 2002;25(6):384-390. 
APPENDICIES 


\section{APPENDIX A}

\section{THE PROBLEM}

\section{Research Question}

Ankle sprains are very common among the athletic population. Cameron et al. ${ }^{2}$ found an injury rate of 33.89 injuries per 1000 person-years at risk across the 4 branches of the United States military. ${ }^{45}$ This injury rate was 5 times more than in civilian studies. ${ }^{2}$ Waterman et al. ${ }^{1}$ found an incidence of 2.15 per 1000 person-years in the United States. Most of the ankle sprains occurred in 15 to 19 year olds and about half of these occurred during athletic activity. A study on youth soccer teams in England found a median injury rate of 0.342 ankle injuries per 1000 hours of exposure, about $13 \%$ of the players. ${ }^{4}$ The injury rate was higher when in competition and also increased with age group. ${ }^{4}$ Over a seven year period, Eskstrand et al. ${ }^{3}$ found a total of 8 ankle injuries per 1000 hours of exposure in professional soccer players. Ankle injures consisted of about $14 \%$ of total injuries reported. ${ }^{3}$ Beynnon et al..$^{5}$ reported a first time injury rate of 0.85 first time ankle sprains per 1000 person-days of sport exposure. The sports evaluated were soccer, basketball, lacrosse, and field hockey. As in many of the reports reviewed women showed a higher incidence of ankle sprains than men. ${ }^{1-5}$

Ankle sprains are usually associated with pain, decreased range of motion, decreased strength, and increased swelling. ${ }^{11}$ These deficits can cause functional and mechanical dysfunction to the ankle joint. Re-injury is common and time lost can be significant. An ankle sprain may develop into chronic ankle instability with re-injury and can cause chronic pain, swelling and instability. 8,11 , Chronic ankle instability is associated with pain, swelling, locking, and stiffness. ${ }^{7}$ There are two causes of chronic ankle 
instability. These are mechanical and functional instability. Mechanical instability is caused by ligamentous laxity, synovial changes, or improper joint kinematics. Functional instabilities result from changes to the stabilizing muscles and have more to do with proprioception. ${ }^{7}$ Hubbard et al. ${ }^{12}$ found that both anterior and inversion joint displacement was still present after 8 weeks of recovery. This lack of recovery could be the cause for further injury that may lead to chronic ankle instability. Typical treatment of ankle sprains often involved non-surgical techniques. Non-surgical treatment included balance training, strengthening, range of motion (ROM) exercises, immobilization, taping, bracing, and proprioceptive exercises. ${ }^{46}$

Joint mobilization decreases pain and increases ROM, strength, proprioception, and balance. ${ }^{19,} 20$ These benefits help to increase joint function and the patients' perception of function. There are many types of joint mobilizations that can be used on the ankle, which include mobilization with movement (MWM) or Mulligan's, Maitland mobilizations, and manipulations. As with other types of treatments there are discrepancies as to the specific use and application of this treatment. ${ }^{19}$ The treatment parameters can include time, direction of mobilization, force used, repetitions, sets, frequency of treatment sessions and length of rest between sessions. Along with the type of mobilization used these parameters can affect the outcome of he treatment. Another variation with joint mobilizations is the treatment benefits/outcomes. Each patient may experience different outcomes for any given treatment.

Most of the benefits or outcomes examined in previous studies have worked to decrease functional instabilities, focusing on proprioception and balance measures. Recently more research has provided a focus on the improvement of mechanical 
instabilities to prevent and properly rehabilitate ankle sprains. One of the ways to improve the mechanical instability deficits is to perform joint mobilizations to increase range of motion.

Research Questions

1. How does joint mobilization affect mechanical instability of the ankle including ROM, patient perception pain, and joint mobility?

2. How effective is joint mobilization on causing these benefits?

3. Do different types of joint mobilizations (Maitland, MWM, and joint manipulations show a difference in the outcome?

Experimental Hypothesis

1. The use of joint mobilization will cause a change, such as an increase in range of motion and a decrease in pain in the ankle following a lateral ankle sprain.

2. Treatment type used for mobilizations will show a difference in treatment outcomes.

3. There will be enough information for each study to be scored by the PEDro scale.

Assumptions

1. All studies will meet all inclusion criteria. Inclusion criteria will include:

a. All studies will be written or translated into English

b. All studies will be related to joint mobilization and ankle sprains

2. No studies will meet exclusion criteria

3. Reviewers using the PEDro scale will be reliable 
Delimitations

1. Research used in this study will only be applied to the ankle joint and joint mobilizations

2. Only studies in the English language will be used.

Operational Definitions

1. Anterior talofibular ligament - A ligament found on the lateral side of the ankle connecting the fibula to the talus. ${ }^{8}$

2. Calcaneofibular ligament - A ligament found on the lateral side of the ankle connecting the fibula to the calcaneus. ${ }^{8}$

3. Chronic ankle instability - Repetitive and recurring ankle sprains that happens over a long period of time, usually causing a decrease in ROM and function. 7,13

4. Effect size - an objective and standardized measure of the magnitude of an observed effect. Calculated by taking the mean difference of experimental and control group and dividing it by the standard deviation of the control group. ${ }^{25}$

5. Functional ankle instability - Joint instability caused by deficits in proprioception and neuromuscular deficits. ${ }^{8,7}$

6. Joint mobilization - A manual therapeutic technique that involves the examiner rhythmically moving the joint through normal joint play. ${ }^{35,46}$

7. Joint manipulation - A manual therapeutic technique that involves the examiner providing a thrust or cavitation to the joint. 28,33

8. Lateral ankle sprain - A stretching or tearing of the ligaments on the lateral side of the ankle, typically the anterior talofibular ligament or calcaneofibular ligament. ${ }^{7}$

9. Maitland mobilizations: Joint mobilizations graded I-IV with various forces applied through different ROM. ${ }^{46}$

10. Mechanical ankle instability - Joint instability caused by deficits anatomical changes such as arthrokinematics, ligamentous structures, muscular changes, and synovial changes. ${ }^{7,8}$ 
11. Mulligan's mobilization or Mobilization with movement (MWM) - Joint mobilizations where force is applied while movement occurs with weight bearing or non-weight bearing, 19,20

12. PEDro scale - a 11 item tool that evaluates the methodological quality of a controlled trial. ${ }^{12,15}$

\section{Limitations}

1. Interpretation of the PEDro scale may be different.

2. Only English or English translated articles will be included.

Significance of the Study

Ankle sprains are a common problem among the athletic population. Pain, swelling, decrease in range of motion, and decrease in strength can inhibit return to full participation. Manual therapy techniques are often used to treat various injuries. Recently athletic trainers have placed greater emphasis on the use of manual therapy. However once the practice has occurred the benefits of joint mobilizations increase the mechanical range of motion available as well as decrease the pain felt by the athlete. To increase use and provide the best treatment for the athlete, evidence based research is needed. This study examined the methodological quality of research studies that concentrate on joint mobilizations for ankle injuries. This study also examined types of mobilization that may affect the outcomes of the patients. This will help athletic trainers to consider the use of joint mobilizations on athletes and help to enhance the healing cascade. This systematic review provided a summary of the information found so that the athletic trainer or other clinician may have a concise analysis of current evidence based research while also evaluating the potential use in the clinical setting. 
This study can be disseminated both through publications and presentations at professional conventions or symposiums. As ankle sprains and manual therapy techniques are often topics discussed at professional meetings, this could be included along with similar topics being presented. Different academic journals would also be interested in circulating this topic to its readers. A few of these journals could be Journal of Athletic Training, American Journal of Sports Medicine, and Physical Therapy in Sport. 


\section{APPENDIX B}

\section{LITERATURE REVIEW}

Introduction

Ankle injuries are a very common occurrence. The most common injury that occurs at the ankle is a lateral ankle sprain. Often if repetitive injury occurs the development of chronic ankle instability occurs. It is important for the clinician to understand the anatomy of ankle sprains to properly treat and rehabilitate this injury. While many studies have evaluated ankle sprains in the general population, it is important to consider the increased demands that the athletic population would place on this joint. Manual therapy techniques are often used to treat various injuries. Athletic trainers have recently placed greater emphasis on the use of these techniques. Joint mobilization techniques are used on a variety of injuries to help with pain control and to increase range of motion. This review aims to provide general information on lateral ankle sprains and chronic ankle instability with a focus on the use of joint mobilizations, mobilizations with movement, and joint manipulations.

Ankle Anatomy

There are three major components that prevent ankle instability. They are the articular surface of the joint, ligaments (static restraint), and musculotendinous units (dynamic restraint)..$^{7,8}$ Two joints, the talocrural joint and the subtalar joint, are typically involved in ankle sprains. The talocrural joint allows for internal and external rotation of the leg and pronation and supination of the foot. ${ }^{8,13}$ It is an articulation between the talus, medial malleolus (tibia), tibial plafond, and lateral malleolus (fibula). In the closed kinetic chain, dorsiflexion occurs when the tibia moves anteriorly on the talus. ${ }^{8,13}$ Ligaments that 
affect the talocrural joint include the anterior talofibular ligament, posterior talofibular ligament, calcaneofibular ligament, and the deltoid ligament. The anterior talofibular ligament, posterior talofibular ligament and calcaneofibular ligament are on the lateral side and therefore are often damaged with the lateral ankle sprain. 7,8,11,13 The deltoid ligament is on the medial side. The anterior talofibular ligament originates on the dorsolateral aspect of the foot and attaches on the lateral malleolus. This ligament prevents the anterior displacement and excessive inversion and internal rotation. 7,8,11,13 Calcaneofibular ligament originates from the lateral malleolus posteriorly and inferiorly to the calcaneus. It prevents excessive supination of the talocrural and subtalar joints. ${ }^{7,8}$ The posterior talofibular ligament originates posteriorly on the lateral malleolus to the posterior lateral section of the talus. It prevents inversion and internal rotation when weight bearing.7, 8

The subtalar joint is formed by the articulation of the talus and the calcaneus. It also allows for internal and external rotation of the leg and pronation and supination of the foot. ${ }^{8}$ The ligamentous support is not well understood but is believed to be controlled with the cervical ligament, interosseous ligament, inferior extensor retinacula, calcaneofibular ligament, lateral talocalcaneal ligament, and fibulotalocalcaneal ligament.7 ${ }^{7}$ The cervical ligament prevents supination and the calcaneofibular ligament prevents excessive inversion and internal rotation of the calcaneus. ${ }^{8}$

The anatomy of the talocrural and subtalar joint is important to understand in order to comprehend the treatment of the ankle sprain. The anatomy is especially important to understand if joint mobilizations are to be used as part of the rehabilitation and treatment. 


\section{Epidemiology}

Ankle sprains are a common injury that affects both the athletic and sedentary populations. For the normal population ankle sprains have been reported at a rate of 2.15 per 1000 person-years with a peak of 7.2 per 1000 person-years in the 15 to 19 age group. ${ }^{1}$ Half of the injuries found in this report occurred during athletic activity with basketball, football and soccer having the highest report of injury. ${ }^{1}$ Females were found to have about 49.7\% of injuries while males accounted for $50.3 \%$.

In contrast to the normal population in the United States, the US military requires personnel to be highly active. Cameron et al. $^{2}$ reported an injury rate of 33.89 injuries per 1000 person-years for all 4 branches of the military. The injury rates were the highest in the under 20 age group with a sharp decline in the 20-24 age group and after age 24 the injury rate decreased steadily with age. ${ }^{2}$ The average injury rate for males was 33.89 injuries per 1000 person-years and for females 41.17 injuries per person-years. ${ }^{2}$ Females that were in the Marines were about twice as likely to get an ankle sprain than men. In the Army females were 68\%, while in-the Navy they were $67 \%$ more likely to sprain their ankle. ${ }^{2}$ In contrast, females in the Air Force were just as likely to get an ankle sprain as males and in some age groups were lower than others. Ankle sprains within the military were from participation in organized and recreational sports, exercise, and physical training. ${ }^{2}$ In a similar study an injury rate of 58.4 per 1000 person-years was found at a United States Military Academy. Women in this study had an increased injury rate ratio of 1.83. About $64.1 \%$ of these sprains occurred during athletics. The sports that reported the highest incidence of injury were men's rugby, women's cheerleading, men's/women's basketball, soccer and lacrosse. 45 
In other countries, ankle sprains are quite common in soccer. Three youth soccer teams in England were examined between August 2007 and July 2008.4 Out of a total of 419 athletes, aged 9-18, 56 ankle injuries occurred that caused a loss of a minimum of 48 hours. ${ }^{4}$ The median injury incidence rate was 0.342 ankle injuries per $1000 \mathrm{~h}$ exposure with 0.862 per 1000 hours of game exposure and 0.077 per 1000 hours of practice exposure. ${ }^{4}$ A median of 16 days were lost per ankle injury. ${ }^{4}$ The general injury trend was an increasing incidence of injury with age group. This was statistically significant for match exposure but not for training exposure. ${ }^{4}$ Seventy-one percent of these ankle injuries were sprains. ${ }^{4}$ About $43.9 \%$ of these injuries occurred in competition. ${ }^{4}$ The annual injury rate was $13 \%$ of the players. ${ }^{4}$ Ekstrand et al. ${ }^{3}$ examined the incidence of injuries in 23 professional soccer teams in the union of European football associations during 2001 through 2008. They found an incidence of 8 injures per $1000 \mathrm{~h}$ of exposure. ${ }^{3}$ Injury incidence was higher during matches than in training. Ankle injures consisted of 625 or about $14 \%$ of injures recorded with most causing a loss of 8-28 days. ${ }^{3}$

Athletic trainers work with a variety of different types of patients. Traditionally athletic trainers have worked with an athletic population aged 14-22 in the high school and collegiate settings. It is important to note that in Cloke et al..$^{4}$ ankle injuries generally increased as the age group increased to 18 years old and in Cameron et al. ${ }^{2}$ and Watermen et al. ${ }^{45}$. incidence of ankle injuries were the highest in age groups under 20 years old. In both Cameron et al. ${ }^{2}$ and Watermen et al. ${ }^{45}$ most injuries sustained were a result of athletic participation. The high volume and incidence of ankle sprains shows the importance of properly understanding the mechanism that lead to ankle sprains and proper treatment of them. While the only sports examined for incidence of ankle sprains was soccer, in both 
cases there was a higher rate of injury during games than practices. This trend is likely to continue due to the higher intensity of play that usually occurs during a game. More research should be conducted regarding incidence of different injuries in sports in the United States to see if they continue to follow these injury trends.

Etiology of the Lateral Ankle Sprain

Ankle sprains are a common injury suffered by both the general and athletic population. Common risk factors for an ankle sprain are a history of previous ankle sprains, no use of a brace or taping, no static stretching or dynamic stretching/warm up before activity, abnormal dorsiflexion range of motion, and no balance or proprioceptive training. $9,13,43,48$ Structural predispositions that may put the athlete at risk for an ankle sprain are increased tibial varum, and non-pathologic talar tilt. ${ }^{9}$ Functional predispositions that may put the athlete at risk include poor postural-control performance, ${ }^{8,9}$ impaired proprioception, ${ }^{8,9}$ and higher eversion to inversion and plantar-dorsiflexion strength ratios. ${ }^{8,9}$ Studies have shown that proprioceptive and neuromuscular training is effective in reducing the number of ankle sprains in athletes. ${ }^{9,10,13}$

The lateral ankle sprain is caused by an inversion and internal rotation force at the ankle joint.8,10,11,This motion puts stress over the lateral ankle ligaments and causes either a stretching or tearing of the ligaments. ${ }^{11}$ The AFTL is typically the first ligament damaged followed by the CFL. $7,8,10,13$ When damaged the ATFL causes transverse plan motion that will produce stress on the surrounding ligaments. Hubbard et al. ${ }^{13}$ conducted a study where after 8 weeks post injury, both anterior and inversion laxity still persisted. While the study did not examine a time frame any longer than 8 weeks, these results could be a cause 
for a patient to develop chronic ankle instability or put them at risk for a second ankle sprain.

Ankle sprains are typically characterized by swelling, pain, bruising over the lateral ankle, decreased range of motion, walking with a limp or loss of function, reduced strength, and joint laxity.8-10 If not healed properly patients can experience some residual symptoms including pain, instability, crepitus, weakness, stiffness, trouble balancing, and swelling. ${ }^{9}$ Most sprains, including ankle sprains, are graded on a scale of I-III. A grade I ankle sprain usually involves no or little loss of function, ligamentous laxity, hemorrhaging, and point tenderness, with decreased range of motion and slight swelling. ${ }^{9,10} \mathrm{~A}$ grade II ankle sprain typically involves some loss of function, a positive anterior drawer test, negative talar tilt test, hemorrhaging, point tenderness, decreased total ankle motion, and swelling. ${ }^{9}, 10,11,50 \mathrm{~A}$ grade III ankle sprain involves almost total loss of function, positive anterior drawer and talar tilt tests, hemorrhaging, extreme point tenderness, decreased total ankle range of motion, and severe swelling. ${ }^{9}, 10$ An evaluation of the lateral ankle sprain typically examines ligament laxity, hemorrhaging, swelling, tenderness, range of motion, strength, and functional testing. ${ }^{9}, 11$ Proper evaluation is needed to determine that the patient is experiencing a lateral ankle sprain and not an injury to other surrounding structures. Some of these patients may need to be referred for imaging to rule out more serious conditions. Chronic Ankle Instability

Chronic ankle instability (CAI) can be caused by functional instability, mechanical instability or a combination of the two. It is a condition in which repeated ankle sprains occur due to these instabilities. ${ }^{8-10,13}$ Those patients who do have repeated ankle sprains and instability are typically diagnosed with CAI. Risk factors for ankle instability are 
increased talar curvature, no use of brace or taping, and no balance or proprioceptive training after acute lateral ankle sprain. ${ }^{9}$ Symptoms and signs of chronic ankle instability can be more subtle than acute lateral ankle sprains. They typically include symptoms similar to a lateral ankle sprain but the ligament laxity will be more obvious and the swelling more subtle.

Functional instability consists of proprioceptive and neuromuscular deficits that cause joint instability. Insufficiencies that cause functional instability include problems with proprioception, neuromuscular control, postural control, or strength.7, 8,13

Proprioceptive deficits have been shown to be altered in patients with CAI and is not fully understood. ${ }^{8}$ Impaired neuromuscular control are present in the injured ankle but these impairments could also be found in the uninjured ankle of the same patient. ${ }^{8}$ Impaired postural control has shown problems with balancing where patients use more hip motion to control the body than ankle motion as is normal. ${ }^{8}$ Strength deficits have been found for eversion and inversion while some patients have also shown no deficits. ${ }^{8}$

There are a few questionnaires useful to determine if the patient has a functional instability. The Cumberland Ankle Instability Tool can be useful in determining the severity of functional ankle instability. It is a 9 item questionnaire and was shown to have a sensitivity of 82.9 and specificity of 74.7 in differentiating between individuals with functional ankle instability. ${ }^{9}$ The Ankle Instability Instrument is a 12 item questionnaire. The overall test-retest reliability was 0.95 for the overall test. ${ }^{9}$ These questionnaires assist the clinician in obtaining the pertinent history, signs, and symptoms that may be present with functional ankle instability. All these evaluation tools are self-reported so there may be slight error if the patient is not honest. 
Mechanical instability is an anatomic change in an initial ankle sprain that causes instability in the ankle. Mechanical instabilities can be caused by pathologic laxity, ${ }^{7}$ impaired arthrokinematics, synovial inflammation ${ }^{7}$ and impingement or degenerative changes.7, 8, 13, Pathologic laxity present depends on the severity of ankle sprain and ligament damage present. ${ }^{12}$ Laxity may be assessed through physical examination, stress radiography, or instrumented arthrometry.7,8,13 The most common ligaments effected are the ATFL and the CFL. Arthorkinematic changes are often due to an anteriorly and inferiorly displaced distal fibula.8,13 When displaced the ATFL will be slack when resting. This change restricts dorsiflexion ROM and as a result can predispose the athlete to lateral ankle sprains. ${ }^{8,13}$ The restricted dorsiflexion doesn't allow the joint to be in the most closed packed position which will allow for the ankle to invert and internally rotate more easily. ${ }^{8}$, ${ }^{15}$ Synovial joint inflammation will also cause ankle instability and reports of pain from subjects. ${ }^{8}$ An individual with mechanical instability has been found to exhibit greater frontal plane displacement and maximum eversion, as well as decreased sagittal plane displacement and maximum plantarflexion during tasks such as walking, steps, running, and jumps. ${ }^{15}$ Caputo et al. also found an increase in anterior translation, internal rotation, and superior translation of the talus in ATFL deficient ankles. These increases in frontal plane displacement can cause degenerative changes and place the patient at risk for more injury. Increasing dorsiflexion ROM and allowing the ankle to be placed in a closed packed position will help to create a more stable joint. Mechanical instability is assessed though physical examination and imaging such as x-rays or MRI. 
Treatment of Ankle Sprains

Typical conservative treatment of ankle sprains includes rest, elevation, compression, ice, and exercise. There have been various findings regarding the effectiveness of rest, ice, compression, and elevation (RICE) in the treatment of ankle sprains. ${ }^{11,49}$ Early treatment and rehabilitation has shown to improve ankle function and a quicker return to participation. 9-10,51 Use of exercises has also been associated with a decrease in re-injury rates. ${ }^{7,9,51}$ However Hing . et. al. ${ }^{23}$ found that early treatment of RICE (rest, ice, compression, elevation) when compared to RICE with therapeutic exercise had similar outcomes up to 11 days after initial injury. ${ }^{23}$ It would be important to evaluate this treatment for a longer outcome to see if a faster return of function occurred. Following emergency department diagnosis, the use of early manual therapy was shown to decrease swelling and pain. ${ }^{11,23}$ The manual therapy used in this study was one of the following: soft tissue mobilization, joint mobilization, isometric mobilization, contract/relax, positional release, or lymphatic drainage. ${ }^{9}$ Another study ${ }^{16}$ examined if immediate physical therapy intervention would provide better outcomes. Initiation of physical therapy within 14 days of diagnosis had significant improvements in Foot and Ankle Outcome Score (FAOS) and visual analog scale (VAS). The use of psychological factors specifically mental relaxation and imagery interventions in addition to typical rehabilitation for patients with ankle sprains has been shown to increase dorsiflexion after intervention. ${ }^{7}$

Conservative treatment must progress as the patient's ankle progresses and heals. The specific treatment of each injury should be individualized and will depend on the severity. In the acute phase, the patient will usually use crutches and progressively bear weight as tolerated. Ankle mobilization as tolerated has been shown to provide an earlier 
return to participation by an average of 4.6 days when compared to immobilization. NATA Manual therapy should be used to reduce swelling, improve pain-free motion, and normalize gait parameters. The manual therapy techniques that can be used in the early phases include lymphatic drainage, active and passive tissue and joint mobilization, and anterior-posterior talar mobilization. ${ }^{9,11}$ In the advanced phase of treatment, manual therapy such as graded joint mobilizations, manipulations, and mobilizations with movement can be used to help improve ankle dorsiflexion. ${ }^{11}$ In addition to manual therapy the used of modalities can also help to decrease pain and swelling. Cryotherapy, 9,11 diathermy, ${ }^{9}$ electrotherapy, ${ }^{9.11}$ low-level laser therapy, ${ }^{9}$ and ultrasound ${ }^{9,11}$ would all be beneficial when treating a lateral ankle sprain both in the acute and progressive phases. Hyperbaric oxygen and biomechanical muscle stimulation (BMS) showed a small effect size but showed benefits for dorsiflexion. ${ }^{50}$ It is also important to note that the use of external support such as a brace or tape has been shown to preventively reduce rates of ankle sprains. ${ }^{9,11,47}$

Normal therapeutic exercises should be used and progressed as tolerated by the patient.9,11 Exercises in the early phases should focus on improving range of motion, decreasing pain, and light strengthening ${ }^{11}$. Static-stretching interventions with home exercise program (HEP) had the strongest effect for increasing dorsiflexion ROM after an acute ankle sprain. ${ }^{52}$ Due to the theory of an anterior displacement of the distal fibula causing increased laxity and pain, mobilization techniques and/or taping techniques could be utilized to reduce this. ${ }^{11}$ Exercises should focus on both eccentric and concentric strengthening. Eccentric strengthening of the ankle after lateral ankle sprain has been shown to improve concentric levels as well. ${ }^{52}$ Exercises in the later phases should focus on 
heavy strengthening, improving coordination, postural control, and proprioception. ${ }^{11}$ All exercises should provide a functional component.11 Studies have shown some decrease in the incidence or re-sprain with the addition of balance and proprioceptive exercises. ${ }^{9}, 11,47$ Balance training used as a preventative measure has been shown to reduce rates of ankle injury.9,11,35 In the advanced phases it is especially important to include sport specific exercises to better prepare the patient for full return to participation. The use of strengthening, balance and functional/sport specific have been shown to help improve postural control, function, coordination, and reduced risk of re-injury.9, 11,47 If conservative treatment fails or injury is not following the proper healing timeline, more invasive techniques can be used. These are either the use of an injection or surgery. Injections of hyaluronic acid combined with rest, ice, elevation, and compression showed reduced pain, more rapid return to participation, and fewer recurrent ankle sprains. ${ }^{10,13}$ The best surgical technique is not clear. Typically with a surgical repair, the anatomy and mechanics are repaired to help recreate normal movement. ${ }^{10,13}$ When surgical treatment and functional rehabilitation treatment was compared at long term follow up, it was found that both were able to reach pre-injuries levels and stress radiographs showed no difference between groups. ${ }^{9}$ Surgery did show a decrease in re-injury rates but had a potential for increased osteoarthritis. ${ }^{9}, 47$ It was also found that while both treatments have similar outcomes. The non-surgical group often had an increased rate of re-injury in the future. ${ }^{47}$ A comprehensive rehabilitation program is needed to modify the adaptations that can occur during the healing process. This rehabilitation should include proprioceptive, neuromuscular, balance, range of motion, and strength training. Often these are targeted in a normal ankle rehabilitation program but at times it can be hard to gain back normal 
dorsiflexion range of motion. The use of joint mobilizations can assist with this and may in combination with the rehabilitation program help to decrease recurrent ankle sprains. ${ }^{17}$ Joint Mobilization

All joint mobilization studies showed improvements from the use of joint mobilizations with no negative effects reported. ${ }^{17-19,26-41,51,53}$ These improvements included increased dorsiflexion, decreased pain levels, and increased function. Maitland mobilizations are typically graded I-IV and are used usually at the peripheral joints. ${ }^{47}$ Maitland joint mobilizations use different velocities of passive joint oscillations through specific arthrokinematic range to achieve therapeutic effects. ${ }^{35} \mathrm{~A}$ grade I mobilization is a small amplitude movement at the beginning of the range of motion. A grade II mobilization is a large amplitude movement at the mid-range of the joint. A grade III is a large amplitude movement at the mid-range of the joint. A grade IV mobilization is a small amplitude movement at the end range of motion. These mobilizations are typically used in combination of the concave-convex rule. When a convex surface moves on a stable concave surface the motion of the convex articulating surface occurs in the opposite direction of movement. Therefore, if trying to mobilize a convex surface on a concave surface, the therapist should provide mobilizations in the opposite direction. The opposite is true when moving a concave surface on a convex surface. Another type of mobilization commonly used is the mobilization with movement (MWM) or the Mulligan's mobilizations. These mobilizations can be used non-weight bearing and weight bearing and involve some type of movement at the joint while pressure is applied. MWM includes a combination of active and passive joint movement throughout ROM with a gliding force. $19-20,42$ 
When joint mobilizations are combined with a HEP it has been shown to improve FAAM-ADL, FAAM-Sport, and pain rating scales after a 4 week intervention. ${ }^{17}$ These improvements present at 4 weeks post injury and at 6 months post injury. ${ }^{17}$ Houston et al. ${ }^{43}$ did a similar study and found that after 1 week of joint mobilizations and traction the FAAM-ADL, and FAAM- Sport both improved. Dynamic Dorsiflexion was also found to decrease significantly immediately after joint mutilations. The average decrease was about 2.98 degrees..$^{18}$ This study implied that the initial effect of joint mobilization is mechanical. MWM has been shown to improve dorsiflexion ROM immediately after treatment. However a change in weight bearing pressure and thermal pain threshold were not found. ${ }^{29}$ In a systematic review, Hoch et al. ${ }^{20}$ noted that after a single treatment dorsiflexion range of motion increased. This increase ranged from 16\%-26\%. ${ }^{20}$ MWM in weight bearing and non-weight bearing was performed on patients with recurrent ankle sprains and found to have initial improvements in posterior glide (WB: 55\%, NWB: 50\%) when compared to pretest. ${ }^{40}$ Weight bearing dorsiflexion range of motion was improved by $26 \%$ when compared to pre-treatment measurements. ${ }^{40}$ The change in posterior talar glide was correlated to the change in weight bearing dorsiflexion only after weight bearing MWM technique was applied. ${ }^{40}$ Overall studies have found an increase in dorsiflexion range of motion, posterior glide, FAAM-ADL, and FAAM-Sport scores.

Hing et al. ${ }^{19}$ performed a systematic review of Mulligan's mobilizations or MWM and described the various parameters used with MWM. Tenets of MWM are accessory glide, physiological movement, pain alteration, immediate effect, over pressure, repetitions, frequency, amount of force, and rest periods. Outcomes assessed were long lasting, client specific outcome measures (CSOM), and overall efficacy of MWM. Accessory glide should be 
at a right angle to the joint or follow the concave-convex rule. All studies reviewed clearly described the direction of the glide. ${ }^{19}$ Physiological movement must occur with MWM. For lateral ankle sprains dorsiflexion or inversion movements were used. The rationale examined for which movement to use with MWM for most of the studies, was based on which movement provoked pain. ${ }^{19} \mathrm{MWM}$ is to be pain-free during application however, as practiced in studies it was used as long as pain reduction was found with mobilization. In $86 \%$ of the studies reviewed the "pain-free" application was used. ${ }^{19}$ This study stated that MWM should be performed with at least pain reduction or pain elimination for appropriate technique. The CSOM improved immediately after post-treatment except in temperature pain threshold which has not been found to be effected in any MWM studies evaluated. Overpressure was only used in $24 \%$ of studies reviewed.

Mulligan recommends 3 sets of 10 repetitions for a typical treatment but variations have been used in research. ${ }^{19-20,42}$ About $86 \%$ of articles reviewed stated repetitions and 52\% stated sets. ${ }^{19}$ Most did follow Mulligan's repetitions. In studies reviewed 1-19 sessions were used. One, three, and six sessions were the most common frequencies used. Interval between treatment sessions varied from 24-48 hours. Amount of force was not stated by Mulligan or the studies evaluated by Hing et al..$^{19}$ Only one study measured the force used with a hand held dynamometer. ${ }^{55}$ The results showed that $66 \%$ or $100 \%$ of maximum force resulted in significant gains but this should be further investigated. ${ }^{55}$ Rest periods used was the largest variation between studies and was only stated in about $52 \%$ of those reviewed. Rest period ranged from $30 \mathrm{~min}-2 \mathrm{hr}$ between sets and 15-60 seconds between repetitions. ${ }^{19}$ The most common rest period was 15 seconds between repetitions. The long term effects were only investigated in $43 \%$ of articles reviewed. Follow up period varied 
from 1-52 weeks and the results showed reduction in pain, increase in participation assessment scores, increases in pain-free strength, function, and ROM. ${ }^{19}$ None found negative long-term effects. All studies incorporated a client specific outcome measure (CSOM). These varied between studies but all included either pain levels, strength, ROM or pressure pain threshold. All studies reviewed found positive results with MWM when compared to placebo or control groups. ${ }^{19}$ The most common significant results were increase in strength, reduction in pain levels, increase in pressure pain threshold, improved upper limb tension tests, and overall function improvements when compared with placebo or control groups.

Hoch et al. ${ }^{20}$ conducted a systematic review on the effectiveness of joint mobilizations on CAI. The findings showed that MWM caused an increase in dorsiflexion range of motion and sensorimotor function. The techniques used for CAI are Maitland joint mobilizations (grade I-IV), Mulligan's mobilization with movement, or high-velocity lowamplitude thrust (Maitland Grade V or manipulation). Manipulations incorporated strategic patient position with quick short thrusts over low amplitude. Patient oriented evidence was limited. Studies reviewed showed FAAM-ADL and FAMM-sports improved but they were unable to determine if it has a meaningful effect from the patient perspective. Talocrural joint mobilization showed to improve dorsiflexion range of motion in CAI but evidence is limited to a single treatment. ${ }^{20}$ Recent investigation confirmed this increase in range of motion over multiple sessions with greater gains found. Improvements in arthrokinematics also have mixed findings. Joint changes required additional investigations to determine the type of effect found. With the star excursion balance test increases in anterior, posteriomedial and posteriolateral reach distance have been shown 
after joint mobilizations over a 2 week period. ${ }^{20}$ Manipulation did not show changes in static or dynamic balance. A relationship does exist between ankle dorsiflexion range of motion and measures of dynamic balance using the star excursion balance test and stepdown test. ${ }^{20}$

Of the studies reviewed, most found improvement regardless of the type or technique of joint mobilization examined. All studies evaluated the general population when performing interventions. In addition the average age of the patients examined were around middle age, than the typical population that an athletic trainer would be working with (14-23). While these studies do show the benefits of treatments, more research is needed to evaluate the effectiveness on the younger athletic population. Some studies that compare different joint mobilization techniques and parameters would also be practical to determine the best technique to use.

Summary

Ankle sprains are a common injury that affects both the general and athletic population. Once injured the patient is at risk for recurrent ankle sprains or developing CAI. The anatomy of the ankle joint is important to understand for the proper treatment and evaluation of the ankle. Lateral ankle sprains occur from an inversion and internal rotation force at the ankle. This typically injures the ATFL and CFL. If a patient suffers from multiple ankle sprains they are typically diagnosed with chronic ankle instability. Chronic ankle instability is usually caused by a combination of mechanical and functional instabilities. Functional instabilities involve damage or dysfunction to proprioceptive or neuromuscular structures. Mechanical instabilities involve damage or dysfunction to the joint articulation, ligaments, synovial fluid, or cartilage. Typical treatment of a lateral ankle 
sprain includes rest, compression, the use of modalities and manual therapy, and therapeutic exercises. Early treatment has been associated with improved outcomes. Since decreased dorsiflexion is thought to put patients at risk for further ankle sprains it is important to gain full range of motion before returning an athlete to participation. This can be accomplished by using joint mobilizations such as Maitland mobilizations and MWM. 


\section{APPENDIX C}

\section{ADDITIONAL METHODS}

Table C1. Protocol for Determining Studies

1. The studies were written in or translated into English.

2. Lateral ankle sprain, chronic ankle instability, or joint mobilizations were present in the title or subject of the study.

3. Lateral ankle sprain, chronic ankle instability, or joint mobilizations were included in the abstract.

4. The main focus of the study must be joint mobilizations.

5. The study must be an experimental study. 
Table C2. PEDro Scale 21,22

1. Eligibility Criteria were specified.

Yes_ No _

2. Subjects were randomly allocated to groups (in a crossover study, subjects were randomly allocated an order in which treatments were received).

3. Allocation was concealed.

Yes_ No _

Yes _ No _

4. The groups were similar at baseline regarding the most important prognostic indicators.

Yes _ No _

5. There was blinding of all subjects.

Yes_ No _

6. There was blinding of all therapists who administered the therapy. $\quad$ Yes _ No _

7. There was blinding of all assessors who measured at least one key outcome.

Yes_No _

8. Measurements of at least one key outcome were obtained from more than $85 \%$ of the subjects initially allocated to groups. $\quad$ Yes _ No

9. All subjects for whom outcome measurements were available received the treatment or control condition as allocated, or where this was not the case, data for at least one key outcome were analyzed by "intention to treat".

Yes _ No

10. The results of between-group statistical comparisons are reported for at least one key outcome.

Yes_ No _

11. The study provides both point measurements and measurements of variability for at least one key outcome.

Yes _ No _ 
Table C3. Descriptions of PEDro Scale ${ }^{21,22}$

\section{Criterion Description}

All criteria Points are awarded when a criterion is clearly satisfied.

Criterion 1 This is satisfied if the study reports the source of subjects and a list of criteria that determines who was eligible to participate in the study. No points are awarded for this criterion.

Criterion 2 This is satisfied if random allocation was used. The precise method of randomization does not need to be stated. However, procedures of allocation by hospital record number, birth date, alternation etc. , do not satisfy this criterion.

Criterion 3 Concealed allocation means that the person who determines if the subject meet the inclusion criteria was unaware of which group the subject would be placed. A point is awarded for this criteria when the study states that allocation was by sealed opaque envelopes or an "offsite" allocation scheduler was used.

Criterion 4 The study must describe at least one measure of severity of the condition being treated and one key outcome measure at baseline. The rater must be satisfied that the groups; outcomes would not be expected to differ, on the basis of differences in prognostic variables alone, by a clinically significant amount. This is satisfied if baseline data of subjects is presented.

Criteria 4, 7- Key outcomes are considered to be those which provide the primary 11 measure of the effectiveness of the therapy.

Criteria 5-7 Blinding means that the person did not know which group the subject was placed in. Subjects and therapists are only considered to be blind if they would not have been able to distinguish between the treatments applied to each group. With self-report outcomes, the assessor is considered to be blind if the subject was blind.

Criterion 8 This is satisfied if the study states that both the number of subjects initially allocated to groups and the number of subjects from whom key outcome measures were obtained. With outcomes that are measured at multiple times, a key outcome must be measured in more than $85 \%$ of subjects at each point in time. 
Criterion 9 Intention to treat means that the analysis was performed as if subjects received the treatment (or control condition) they were allocated to. This is satisfied if the study states that all subjects received treatment or control conditions as assigned or an intent to treat analysis was found.

Criterion 10 A between-group statistical comparison is a statistical comparison of one group with another. This statistical comparison may involve a comparison of 2 or more treatments or comparison of treatment with a control condition. The analysis may be a comparison of outcomes measured after the treatment was administered or a comparison of the change in one group with the change in another. The comparison may be hypothesis testing or an estimate.

Criterion 11 A point measure is a measure of the size of the treatment effect. The treatment effect may be the difference in group outcomes or the outcome in all groups. Measures of variability include standard deviations, standard errors, confidence intervals, interquartile ranges, and ranges. Point measures and/or measures of variability may be provided graphically. When outcomes are categorical, this is met if the number of subjects in each category is given for each group. 
APPENDIX D

\section{ADDITIONAL RESULTS}

Table D1. Joint Mobilization Studies

\begin{tabular}{|c|c|c|c|c|c|c|}
\hline Study & Participants & Intervention & Outcomes measures & Results & Conclusions & $\begin{array}{l}\text { PEDro } \\
\text { Scale }\end{array}$ \\
\hline $\begin{array}{l}\text { Cosby et } \\
\text { al. }{ }^{30}\end{array}$ & $\begin{array}{l}17 \text { subjects with an } \\
\text { acute lateral ankle } \\
\text { sprain with previous } \\
\text { immobilization in } \\
\text { walking boot; Average } \\
\text { age } 19.76\end{array}$ & $\begin{array}{l}\text { Single-blinded, } \\
\text { randomized } \\
\text { control trial; } \\
\text { control group or } \\
\text { talocrural joint } \\
\text { mobilization group } \\
\text { (Maitland grade III } \\
\text { AP); } 1 \text {-30 second } \\
\text { bout }\end{array}$ & $\begin{array}{l}\text { Dorsiflexion ROM (NWB), } \\
\text { Posterior Talar translation, } \\
\text { Self-reported function }\end{array}$ & $\begin{array}{l}\text { Dorsiflexion ROM: No significant } \\
\text { differences by time or group } \\
\text { effect. When combined a } \\
\text { significant improvement in ROM } \\
\text { was found. Posterior Talar } \\
\text { Translation: No significant } \\
\text { difference by time or group effect. }\end{array}$ & $\begin{array}{l}\text { A single treatment of grade III AP } \\
\text { talocrural joint mobilization was not } \\
\text { effective at increasing ankle dorsiflexion } \\
\text { ROM, decreasing posterior talar glide, or } \\
\text { self-reported measures. There was } \\
\text { improvement in dorsiflexion ROM at } 24 \\
\text { hour follow up after removal of walking } \\
\text { boot. This suggests that } 24 \text { hours of } \\
\text { ambulation caused in increase in ROM } \\
\text { and function. }\end{array}$ & $8 / 10$ \\
\hline $\begin{array}{l}\text { Green et } \\
\text { al. }{ }^{32}\end{array}$ & $\begin{array}{l}38 \text { subjects ( } 12 \text { female, } \\
26 \text { male) diagnosed } \\
\text { with acute ankle sprain; } \\
\text { Average age } 25.5\end{array}$ & $\begin{array}{l}\text { Randomized } \\
\text { controlled trial; } \\
\text { control group } \\
\text { (RICE) or } \\
\text { anteroposterior } \\
\text { mobilization } \\
\text { group; } 6 \\
\text { treatments over } 2 \\
\text { week period }\end{array}$ & $\begin{array}{l}\text { Dorsiflexion ROM, Gait (stride } \\
\text { speed, step length, and single } \\
\text { support time) }\end{array}$ & $\begin{array}{l}\text { Dorsiflexion ROM: Experimental } \\
\text { group improved } 4.3 \text { degrees } \\
\text { where the control group improved } \\
0.9 \text { degrees }\end{array}$ & $\begin{array}{l}\text { This study found that anteroposterior } \\
\text { joint mobilizations improved ankle ROM } \\
\text { in dorsiflexion. }\end{array}$ & $7 / 10$ \\
\hline $\begin{array}{l}\text { Hoch et } \\
\text { al. }{ }^{34}\end{array}$ & $\begin{array}{l}20 \text { subjects ( } 11 \text { female, } \\
9 \text { male) with self- } \\
\text { reported CAI; Average } \\
\text { age } 23.4\end{array}$ & $\begin{array}{l}\text { Crossover design, } \\
\text { Joint mobilization } \\
\text { (Maitland grade } \\
\text { III) and control } \\
\text { (rest for } 5 \text { min); } 1 \\
\text { treatment each }\end{array}$ & $\begin{array}{l}\text { Dorsiflexion ROM, Posterior } \\
\text { Talar displacement and } \\
\text { stiffness, SEBT, SLS }\end{array}$ & $\begin{array}{l}\text { Dorsiflexion ROM: A significant } \\
\text { increase was found in dorsiflexion } \\
\text { ROM in the joint mobilization } \\
\text { group. Posterior Talar } \\
\text { Displacement and Stiffness: No } \\
\text { significant difference was found } \\
\text { with posterior talar displacement } \\
\text { or stiffness. }\end{array}$ & $\begin{array}{l}\text { A single treatment of Maitland Grade III } \\
\text { anterior/posterior joint mobilization } \\
\text { effects dorsiflexion ROM and TTB } \\
\text { postural control measures in patients } \\
\text { with CAI. }\end{array}$ & $5 / 10$ \\
\hline $\begin{array}{l}\text { Hoch et } \\
\text { al. } 35\end{array}$ & $\begin{array}{l}12 \text { subjects ( } 6 \text { female, } 6 \\
\text { male) with CAI; } \\
\text { Average age } 27.4\end{array}$ & $\begin{array}{l}\text { Prospective cohort } \\
\text { study; Joint } \\
\text { mobilization } \\
\text { (Maitland Grade } \\
\text { II(traction) and } \\
\text { Maitland }\end{array}$ & Dorsiflexion ROM (WB), SEBT & $\begin{array}{l}\text { Dorsiflexion ROM: A significant } \\
\text { increase was found throughout } \\
\text { the course of the study }\end{array}$ & $\begin{array}{l}\text { A } 2 \text { week joint mobilization intervention } \\
\text { resulted in improvements of } \\
\text { dorsiflexion. This study targeted non- } \\
\text { contractile tissue. }\end{array}$ & $5 / 10$ \\
\hline
\end{tabular}


III(posterior

glide)); 6

treatments over 8

20 days

\begin{tabular}{|c|c|c|c|}
\hline $\begin{array}{l}\text { Yeo et } \\
\text { al. }{ }^{41}\end{array}$ & $\begin{array}{l}13 \text { subjects ( } 3 \text { female, } \\
10 \text { male) with } \\
\text { unilateral sub-acute } \\
\text { supination injury; } \\
\text { Average Age } 29.5\end{array}$ & $\begin{array}{l}\text { Randomized, } \\
\text { double-blind, } \\
\text { repeated measures } \\
\text { study; joint } \\
\text { mobilization } \\
\text { group, manual } \\
\text { contact control } \\
\text { group, and no } \\
\text { contact control } \\
\text { group; } 1 \text { treatment } \\
\text { each with } 48 \text { hour } \\
\text { washout period }\end{array}$ & $\begin{array}{l}\text { Dorsiflexion ROM (WB), } \\
\text { Ankle functional score, Pain } \\
\text { related measures }\end{array}$ \\
\hline
\end{tabular}

Dorsiflexion ROM (WB): A

significant increase of $9.6 \mathrm{~mm}$

occurred after joint mobilization group. Pain related measures:

Pressure pain threshold

significantly increased by $17.76 \%$

following the joint mobilization

condition. VAS scores showed a

slight decrease after joint

mobilization but it was not statistically significant.
This study showed that joint

mobilization using the anterior-

posterior glide technique produced

immediate and rapid onset hypoalgesic

effect and an improvement in ankle

dorsiflexion.

Table D2. Mobilizations with Movement Studies

\begin{tabular}{|c|c|c|c|c|c|c|}
\hline Study & Participants & Intervention & Outcome Measure & Results & Conclusion & $\begin{array}{l}\text { PEDro } \\
\text { Score }\end{array}$ \\
\hline Akre et al. ${ }^{26}$ & $\begin{array}{l}30 \text { subjects (16 } \\
\text { females, } 14 \\
\text { males) with } \\
\text { diagnosis with } \\
\text { unilateral ankle } \\
\text { sprain with } \\
\text { symptoms } \\
\text { lasting longer } \\
\text { than } 7 \text { days; } \\
\text { Average age } \\
25.14\end{array}$ & $\begin{array}{l}\text { Randomized clinical } \\
\text { trial, Mulligan's MWM- } \\
\text { WB group and MWM- } \\
\text { NWB group; } \\
\text { Posteroanterior glide of } \\
\text { tibia; } 10 \text { consecutive } \\
\text { days }\end{array}$ & $\begin{array}{l}\text { VAS, ankle range } \\
\text { of motion, and } \\
\text { Foot and Ankle } \\
\text { Disability Index } \\
\text { (FADI) }\end{array}$ & $\begin{array}{l}\text { VAS: MWM-WB and MWM-NWB } \\
\text { techniques significantly reduced pain. } \\
\text { ROM: MWM-WB and MWM-NWM } \\
\text { significantly improved plantarflexion } \\
\text { ROM. Dorsiflexion ROM was improved in } \\
\text { both treatments as well but was not } \\
\text { statistically significant. }\end{array}$ & $\begin{array}{l}\text { Weight bearing MWM are more effective } \\
\text { than non-weight bearing mobilizations } \\
\text { when treating ankle sprains. }\end{array}$ & $6 / 10$ \\
\hline Collins et al. 29 & $\begin{array}{l}16 \text { ( } 8 \text { females, } 8 \\
\text { males) with } \\
\text { grade II ankle } \\
\text { lateral ligament } \\
\text { sprain sustained } \\
\text { over about } 40 \\
\text { days before } \\
\text { testing; Average } \\
\text { age } 28.25\end{array}$ & $\begin{array}{l}\text { Double Blind Crossover } \\
\text { Design; MWM-WB } \\
\text { (posteroanterior tibial } \\
\text { glide), Placebo } \\
\text { treatment (take up } \\
\text { slack, no mobilization), } \\
\text { and no treatment } \\
\text { control; } 1 \text { treatment }\end{array}$ & $\begin{array}{l}\text { Dorsiflexion ROM } \\
\text { (WB) and Pain } \\
\text { (pressure and } \\
\text { thermal pain } \\
\text { threshold) }\end{array}$ & $\begin{array}{l}\text { Dorsiflexion ROM: MWM-WB was } \\
\text { found to have a significant treatment } \\
\text { effect from pre to post test scores. No } \\
\text { significant finding was found with the } \\
\text { placebo condition. Pain: When grouped } \\
\text { together pain pressure threshold for the } \\
\text { ATFL and TA was found to have a } \\
\text { significant effect over time. A significant } \\
\text { improvement was found in pre and post } \\
\text { test differences for pain pressure } \\
\text { threshold with the placebo group. The } \\
\text { others items tested were found to be } \\
\text { statistically insignificant }\end{array}$ & $\begin{array}{l}\text { MWM-WB significantly increases } \\
\text { talocrural dorsiflexion after application } \\
\text { initially in a subacute ankle sprain. The } \\
\text { lack of significant findings for pain relief } \\
\text { suggest that the effect of this treatment is } \\
\text { more mechanical than hypoalgesic. }\end{array}$ & $7 / 10$ \\
\hline
\end{tabular}


Gilbreath et

al. ${ }^{31}$

11 subjects $(6$

females and 5

males) with self-

reported $\mathrm{CAI}$;

physically

active; Average

age 21.5

Nambi et al. ${ }^{37} \quad 30$ field hockey

players $(5$

female, 10 male)

with lateral

ankle sprain;

Age: 13-17

23 subjects ( 15

female, 8 male)

with unilateral

ankle sprains

within the past 2

years and

restriction in

WB dorsiflexion

Average Age 25

Vicenzino et

16 subjects ( 8

female, 8 male)

with history of

recurrent lateral

ankle sprain and

deficits in

posterior talar

glide and WB

dorsiflexion;

Average age

19.8
Within subject repeated measures design;

MWM-WB (posterior to

anterior pressure on

tibia); 3 tx over 2 week

period

experimental design;

Kinesio tape group and

MWM-WB group

posteroanterior glide

of the tibia); 3

treatments over 1 week

Crossover design with

random assignment;

Sham joint mobilization

group and MWM-WB

group; 1 treatment of

each condition with 1

week as a washout

period

Double blind

randomized crossover

experimental study;

MWM-WB group, MWM-

NWB group, no

treatment control

group; 1 treatment in

each group with 48 hour

washout
Dorsiflexion ROM

(WB), Dynamic

Dorsiflexion ROM: No significant changes were noticed in dorsiflexion ROM.

ADL, FAAM-Sport

Numeric Pain

Rating Scale and

Dorsiflexion ROM

(WB) significantly decreased by $75.9 \%$ in MWM-WB group and 55.69\% in kinesio tape group. Dorsiflexion ROM:

Dorsiflexion range of motion showed a significant increase of $71.07 \%$ in MWMWB group and $27.64 \%$ kinesio tape.

Dorsiflexion ROM Dorsiflexion ROM: A significantly

(WB) greater change in DF ROM was found in the MWM-WB group when compared to the sham group.

Posterior Talar

Glide and

Dorsiflexion ROM

significantly reduced the posterior glide deficit by $50 \%$ for NWB and $55 \%$ for WB. Dorsiflexion ROM: MWM

conditions reduced unaffected side

deficit by $26 \%$ compared to a $9 \%$

reduction with the control condition.
This study found that MWM-WB did not improve with dorsiflexion ROM, dynamic balance, or patient-centered measures of ADL. MWM did improve patient centered measure of sport related activities in individuals with CAI

Both MWM-WB and kinesio tape are effective at reducing pain and improving dorsiflexion ROM in the sub-acute lateral ankle sprain. The subjects in the MWM-

WB group showed better improvement in both pain reduction and dorsiflexion ROM

MWM-WB immediately improves dorsiflexion ROM after treatment.

However this improvement is relatively small even though it is statistically significant.

This study shows the positive effect that ankle sprain. They showed improvements in both posterior talar glide and dorsiflexion ROM 
Table D3. Joint Manipulation Studies

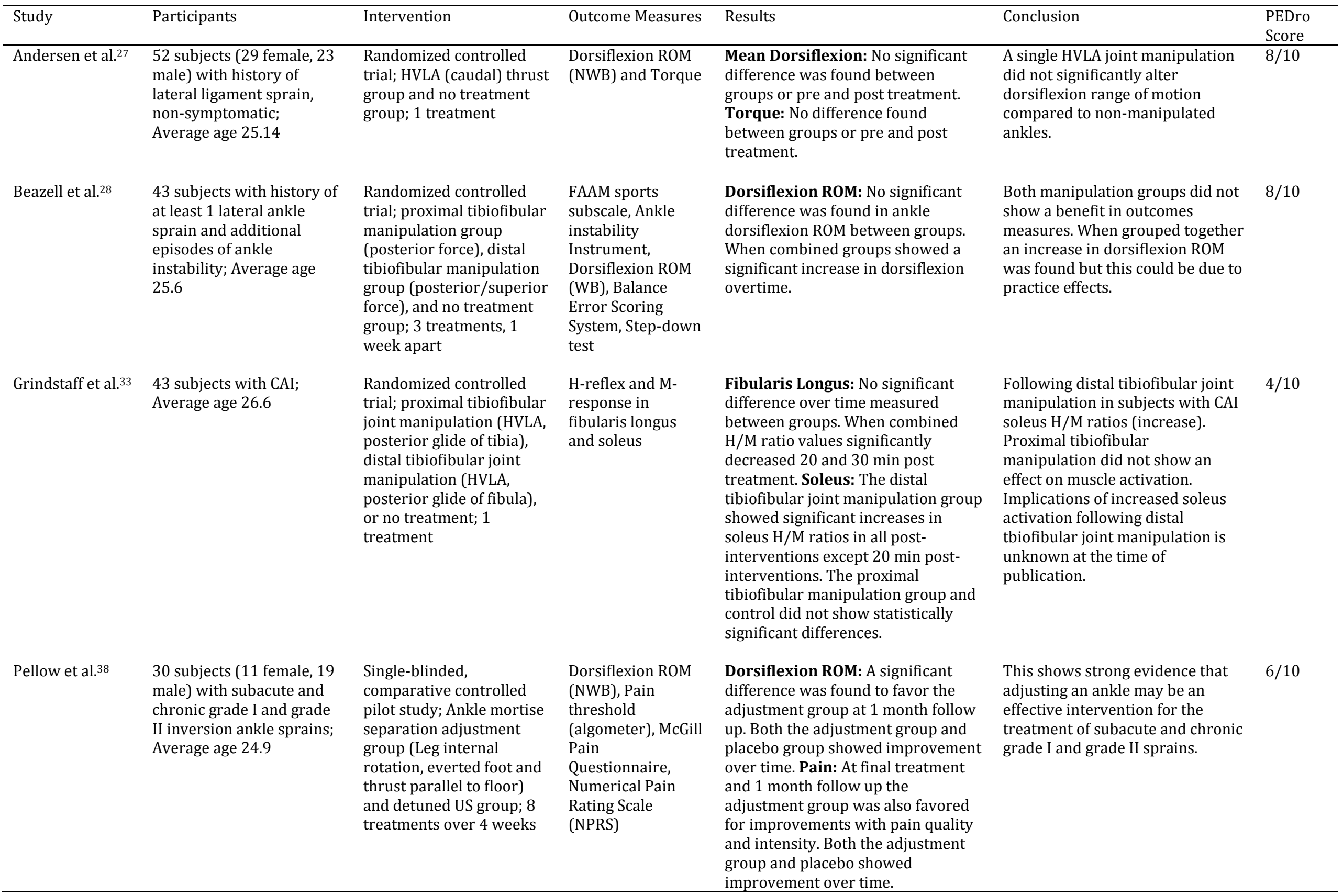


Table D4. Joint Mobilization and Manipulation Study

\begin{tabular}{|c|c|c|c|c|c|c|}
\hline Study & Participants & Intervention & $\begin{array}{l}\text { Outcome } \\
\text { Measures }\end{array}$ & Results & Conclusion & $\begin{array}{l}\text { PEDro } \\
\text { Score }\end{array}$ \\
\hline $\begin{array}{l}\text { Marrón-Gómez } \\
\text { et al.36 }\end{array}$ & $\begin{array}{l}52 \text { Subjects ( } 21 \text { female, } 31 \\
\text { male) with CAI; Average } \\
\text { age } 20.7\end{array}$ & $\begin{array}{l}\text { Randomized controlled } \\
\text { clinical trial; MWM-WB } \\
\text { group (posteroanterior } \\
\text { glide to tibia), HVLA } \\
\text { manipulation group } \\
\text { (caudal force), and } \\
\text { placebo (take up slack of } \\
\text { joint); } 1 \text { treatment }\end{array}$ & $\begin{array}{l}\text { Dorsiflexion } \\
\text { ROM (WB) }\end{array}$ & $\begin{array}{l}\text { Dorsiflexion ROM: Both MWM-WB } \\
\text { and HVLA groups showed a } \\
\text { significant increase in dorsiflexion } \\
\text { ROM when compared to the placebo } \\
\text { but did not show a significant } \\
\text { difference when compared to each } \\
\text { other. The increase in ROM was found } \\
\text { in both within the HVLA group and } \\
\text { MWM-WB group but the MWM-WB } \\
\text { group had a moderate effect size } \\
\text { where HVLA group had a small effect } \\
\text { size. HVLA group had a large effect } \\
\text { when compared to placebo. }\end{array}$ & $\begin{array}{l}\text { A single application of MWM-WB or } \\
\text { HLVA improves ankle dorsiflexion in } \\
\text { people with CAI and last for at least } \\
\text { two days. MWM-WB showed greater } \\
\text { effect sizes than HVLA. }\end{array}$ & $7 / 10$ \\
\hline
\end{tabular}


Table D5. PEDro Results

\begin{tabular}{|c|c|c|c|c|c|c|c|c|c|c|c|c|}
\hline Author & $\begin{array}{l}\text { Criteria } \\
1\end{array}$ & $\begin{array}{l}\text { Criteria } \\
2\end{array}$ & $\begin{array}{l}\text { Criteria } \\
3\end{array}$ & $\begin{array}{l}\text { Criteria } \\
4\end{array}$ & $\begin{array}{l}\text { Criteria } \\
5\end{array}$ & $\begin{array}{l}\text { Criteria } \\
6\end{array}$ & $\begin{array}{l}\text { Criteria } \\
7\end{array}$ & $\begin{array}{l}\text { Criteria } \\
8\end{array}$ & $\begin{array}{l}\text { Criteria } \\
9\end{array}$ & $\begin{array}{l}\text { Criteria } \\
10\end{array}$ & $\begin{array}{l}\text { Criteria } \\
11\end{array}$ & Total \\
\hline Akre et al. ${ }^{26}$ & Y & $\mathrm{Y}$ & $\mathrm{N}$ & $\mathrm{Y}$ & $\mathrm{N}$ & $\mathrm{N}$ & $\mathrm{N}$ & $\mathrm{Y}$ & $\mathrm{Y}$ & $\mathrm{Y}$ & $\mathrm{Y}$ & $6 / 10$ \\
\hline $\begin{array}{l}\text { Beazell et } \\
\text { al. }{ }^{28}\end{array}$ & $\mathrm{~N}$ & Y & Y & Y & $\mathrm{N}$ & $\mathrm{N}$ & Y & Y & Y & Y & Y & $8 / 10$ \\
\hline $\begin{array}{l}\text { Collins et } \\
\text { al. }^{29}\end{array}$ & Y & Y & $\mathrm{N}$ & $\mathrm{Y}$ & Y & $\mathrm{N}$ & $\mathrm{Y}$ & $\mathrm{Y}$ & $\mathrm{N}$ & $\mathrm{Y}$ & $\mathrm{Y}$ & $7 / 10$ \\
\hline $\begin{array}{l}\text { Gilbreath et } \\
\text { al.31 }\end{array}$ & $\mathrm{Y}$ & $\mathrm{N}$ & $\mathrm{N}$ & $\mathrm{N}$ & $\mathrm{N}$ & $\mathrm{N}$ & $\mathrm{N}$ & $\mathrm{N}$ & $\mathrm{N}$ & $\mathrm{N}$ & Y & $1 / 10$ \\
\hline $\begin{array}{l}\text { Green et al. }{ }^{32} \\
\text { Grindstaff et }\end{array}$ & $\mathrm{Y}$ & $\mathrm{Y}$ & $\mathrm{Y}$ & $\mathrm{Y}$ & $\mathrm{N}$ & $\mathrm{N}$ & Y & $\mathrm{Y}$ & $\mathrm{N}$ & $\mathrm{Y}$ & Y & $7 / 10$ \\
\hline $\mathrm{al}^{33}$ & $\mathrm{Y}$ & Y & $\mathrm{N}$ & $\mathrm{Y}$ & $\mathrm{N}$ & $\mathrm{N}$ & $\mathrm{Y}$ & $\mathrm{N}$ & $\mathrm{N}$ & $\mathrm{Y}$ & $\mathrm{N}$ & $4 / 10$ \\
\hline $\begin{array}{l}\text { al. }{ }^{37} \\
\text { Pellow et }\end{array}$ & Y & $\mathrm{N}$ & $\mathrm{N}$ & $\mathrm{Y}$ & $\mathrm{N}$ & $\mathrm{N}$ & $\mathrm{N}$ & $\mathrm{N}$ & $\mathrm{N}$ & $\mathrm{Y}$ & Y & $3 / 10$ \\
\hline $\mathrm{al}^{38}$ & Y & $\mathrm{Y}$ & $\mathrm{N}$ & $\mathrm{N}$ & $\mathrm{N}$ & $\mathrm{N}$ & $\mathrm{Y}$ & $\mathrm{Y}$ & $\mathrm{Y}$ & $\mathrm{Y}$ & $\mathrm{Y}$ & $6 / 10$ \\
\hline $\begin{array}{l}\text { Reid et al. }{ }^{39} \\
\text { Vicenzino et }\end{array}$ & Y & Y & $\mathrm{N}$ & $\mathrm{Y}$ & $\mathrm{N}$ & $\mathrm{N}$ & Y & $\mathrm{Y}$ & $\mathrm{Y}$ & $\mathrm{Y}$ & Y & $7 / 10$ \\
\hline al. ${ }^{40}$ & $\mathrm{Y}$ & Y & $\mathrm{N}$ & $\mathrm{Y}$ & $\mathrm{N}$ & $\mathrm{N}$ & Y & $\mathrm{N}$ & Y & $\mathrm{Y}$ & $\mathrm{Y}$ & $6 / 10$ \\
\hline Yeo et al. ${ }^{41}$ & $\mathrm{Y}$ & $\mathrm{Y}$ & $\mathrm{N}$ & $\mathrm{Y}$ & $\mathrm{N}$ & $\mathrm{N}$ & $\mathrm{Y}$ & $\mathrm{Y}$ & $\mathrm{N}$ & $\mathrm{Y}$ & $\mathrm{N}$ & $5 / 10$ \\
\hline
\end{tabular}


Table D6. Akre et al. ${ }^{26}$ PEDro scale

1. Eligibility criteria were specified

No _ Yes $\underline{X}$

2. Subjects were randomly allocated to groups (in a crossover study, subjects

No_Yes $\underline{X}$ were randomly allocated an order in which treatments were received)

3. Allocation was concealed

No $\underline{X} Y e s$

4. The groups were similar at baseline regarding the most important

No_Yes $\underline{X}$ prognostic indicators

5. There was blinding of all subjects

6. There was blinding of all therapists who administered the therapy

No $\underline{X}$ Yes

7. There was blinding of all assessors who measured at least one key

No $\underline{X}$ Yes

No $\underline{X}$ Yes

8. Measures of at least one key outcome were obtained from more than $85 \%$

No_Yes $\underline{X}$ of the subjects initially allocated to groups

9. All subjects for whom outcome measures were available received the

No_Yes $\underline{X}$ treatment or control condition as allocated or, where this was not the case, data for at least one key outcome was analysed by "intention to treat"

10. The results of between-group statistical comparisons are reported for at $\quad$ No _ Yes $\underline{X}$ least one key outcome

11. The study provides both point measures and measures of variability for at No_Yes $\underline{X}$ least one key outcome

Total: $6 / 10$

Table D7. Andersen et al. ${ }^{27}$ PEDro scale

1. Eligibility criteria were specified

No_Yes $\underline{\mathrm{X}}$

2. Subjects were randomly allocated to groups (in a crossover study, subjects

No_Yes $\underline{X}$

were randomly allocated an order in which treatments were received)

3. Allocation was concealed

No_Yes $\underline{\mathrm{X}}$

4. The groups were similar at baseline regarding the most important

No_Yes $\underline{X}$ prognostic indicators

5. There was blinding of all subjects

No $\underline{\mathrm{X} Y e s}$ -

6. There was blinding of all therapists who administered the therapy

No $\underline{X}$ Yes

7. There was blinding of all assessors who measured at least one key

No_Yes $\underline{X}$

8. Measures of at least one key outcome were obtained from more than $85 \%$

No_Yes $\underline{X}$ of the subjects initially allocated to groups

9. All subjects for whom outcome measures were available received the

No_Yes $\underline{X}$ treatment or control condition as allocated or, where this was not the case, data for at least one key outcome was analysed by "intention to treat"

10. The results of between-group statistical comparisons are reported for at least one key outcome

11. The study provides both point measures and measures of variability for at No_Yes $\underline{X}$ least one key outcome 
Table D8. Beazell et al ${ }^{28}$. PEDro scale

1. Eligibility criteria were specified

No $\underline{X}$ Yes

2. Subjects were randomly allocated to groups (in a crossover study, subjects

No_Yes $\underline{X}$ were randomly allocated an order in which treatments were received)

3. Allocation was concealed

No_Yes $\underline{X}$

4. The groups were similar at baseline regarding the most important

No_Yes $\underline{X}$ prognostic indicators

5. There was blinding of all subjects

No $\underline{X}$ Yes

6. There was blinding of all therapists who administered the therapy

No X Yes

7. There was blinding of all assessors who measured at least one key

No_Yes $\underline{X}$

8. Measures of at least one key outcome were obtained from more than $85 \%$

No_Yes $\underline{X}$ of the subjects initially allocated to groups

9. All subjects for whom outcome measures were available received the

No_Yes $\underline{X}$ treatment or control condition as allocated or, where this was not the case, data for at least one key outcome was analysed by "intention to treat"

10. The results of between-group statistical comparisons are reported for at least one key outcome

11. The study provides both point measures and measures of variability for at No _Yes $\underline{X}$ least one key outcome

Total: $8 / 10$

Table D9. Collins et al. ${ }^{29}$ PEDro scale

1. Eligibility criteria were specified

No_Yes $\underline{\mathrm{X}}$

2. Subjects were randomly allocated to groups (in a crossover study, subjects

No_Yes $\underline{X}$

were randomly allocated an order in which treatments were received)

3. Allocation was concealed

No $\underline{X}$ Yes

4. The groups were similar at baseline regarding the most important

No_Yes $\underline{X}$ prognostic indicators

5. There was blinding of all subjects

No_Yes $\underline{X}$

6. There was blinding of all therapists who administered the therapy

No $\underline{X}$ Yes

7. There was blinding of all assessors who measured at least one key

No_Yes $\underline{X}$

8. Measures of at least one key outcome were obtained from more than $85 \%$

No_Yes $\underline{X}$ of the subjects initially allocated to groups

9. All subjects for whom outcome measures were available received the

No $\underline{X}$ Yes treatment or control condition as allocated or, where this was not the case, data for at least one key outcome was analysed by "intention to treat"

10. The results of between-group statistical comparisons are reported for at least one key outcome

11. The study provides both point measures and measures of variability for at No _ Yes $\underline{X}$ least one key outcome 
Table D10. Cosby et al..$^{30}$ PEDro scale

1. Eligibility criteria were specified

No_Yes $\underline{X}$

2. Subjects were randomly allocated to groups (in a crossover study, subjects

No_Yes $\underline{X}$ were randomly allocated an order in which treatments were received)

3. Allocation was concealed

No_Yes $\underline{X}$

4. The groups were similar at baseline regarding the most important

No_Yes $\underline{X}$ prognostic indicators

5. There was blinding of all subjects

No $\underline{X}$ Yes

6. There was blinding of all therapists who administered the therapy

No X Yes

7. There was blinding of all assessors who measured at least one key

No_Yes $\underline{X}$

8. Measures of at least one key outcome were obtained from more than $85 \%$ of the subjects initially allocated to groups

9. All subjects for whom outcome measures were available received the

No_Yes $\underline{X}$

No_Yes $\underline{X}$ treatment or control condition as allocated or, where this was not the case, data for at least one key outcome was analysed by "intention to treat"

10. The results of between-group statistical comparisons are reported for at least one key outcome

11. The study provides both point measures and measures of variability for at No_Yes $\underline{X}$ least one key outcome

Total: $8 / 10$

Table D11. Gilbreath et al. ${ }^{31}$ PEDro scale

1. Eligibility criteria were specified No_Yes $\underline{\mathrm{X}}$

2. Subjects were randomly allocated to groups (in a crossover study, subjects were randomly allocated an order in which treatments were received)

3. Allocation was concealed

4. The groups were similar at baseline regarding the most important

No $\underline{X}$ Yes prognostic indicators

No $\underline{X} Y e s$

No $\underline{X}$ Yes

5. There was blinding of all subjects

6. There was blinding of all therapists who administered the therapy

7. There was blinding of all assessors who measured at least one key

8. Measures of at least one key outcome were obtained from more than $85 \%$ of the subjects initially allocated to groups

9. All subjects for whom outcome measures were available received the treatment or control condition as allocated or, where this was not the case, data for at least one key outcome was analysed by "intention to treat"

10. The results of between-group statistical comparisons are reported for at least one key outcome

No $\underline{X}$ Yes No $\underline{X}$ Yes No $\underline{X}$ Yes No $\underline{X}$ Yes No $\underline{X}$ Yes No $\underline{X}$ Yes

11. The study provides both point measures and measures of variability for at No _ Yes $\underline{X}$ least one key outcome 
Table D12. Green et al. ${ }^{32}$ PEDro scale

1. Eligibility criteria were specified

No_Yes $\underline{X}$

2. Subjects were randomly allocated to groups (in a crossover study, subjects

No_Yes $\underline{X}$ were randomly allocated an order in which treatments were received)

3. Allocation was concealed

No_Yes $\underline{\mathrm{X}}$

4. The groups were similar at baseline regarding the most important

No_Yes $\underline{X}$ prognostic indicators

5. There was blinding of all subjects

No $\underline{X}$ Yes

6. There was blinding of all therapists who administered the therapy

No $\underline{X}$ Yes

7. There was blinding of all assessors who measured at least one key

No_Yes $\underline{X}$

8. Measures of at least one key outcome were obtained from more than $85 \%$ of the subjects initially allocated to groups

9. All subjects for whom outcome measures were available received the

No_Yes $\underline{X}$

No $\underline{X}$ Yes treatment or control condition as allocated or, where this was not the case, data for at least one key outcome was analysed by "intention to treat"

10. The results of between-group statistical comparisons are reported for at least one key outcome

11. The study provides both point measures and measures of variability for at No_Yes $\underline{X}$ least one key outcome

Total: $7 / 10$

Table D13. Grindstaff et al.33 PEDro scale

1. Eligibility criteria were specified

No_Yes $\underline{X}$

2. Subjects were randomly allocated to groups (in a crossover study, subjects were randomly allocated an order in which treatments were received)

3. Allocation was concealed

4. The groups were similar at baseline regarding the most important

No $\underline{X}$ Yes prognostic indicators

5. There was blinding of all subjects

6. There was blinding of all therapists who administered the therapy

No_Yes $\underline{\mathrm{X}}$

7. There was blinding of all assessors who measured at least one key

No $\underline{X}$ Yes

No $\underline{X}$ Yes

8. Measures of at least one key outcome were obtained from more than $85 \%$

No_Yes $\underline{X}$

of the subjects initially allocated to groups

9. All subjects for whom outcome measures were available received the

No $\underline{X}$ Yes

treatment or control condition as allocated or, where this was not the

No $\underline{X}$ Yes case, data for at least one key outcome was analysed by "intention to treat"

10. The results of between-group statistical comparisons are reported for at least one key outcome

No_Yes $\underline{X}$

11. The study provides both point measures and measures of variability for at No $\underline{X}$ Yes least one key outcome 
Table D14. Hoch et al. ${ }^{34}$ PEDro scale

1. Eligibility criteria were specified

No $\underline{X}$ Yes

2. Subjects were randomly allocated to groups (in a crossover study, subjects

No_Yes $\underline{X}$ were randomly allocated an order in which treatments were received)

3. Allocation was concealed

No_Yes $\underline{X}$

4. The groups were similar at baseline regarding the most important

No $\underline{X}$ Yes prognostic indicators

5. There was blinding of all subjects

No $\underline{X}$ Yes

6. There was blinding of all therapists who administered the therapy

No $\underline{X}$ Yes

7. There was blinding of all assessors who measured at least one key

No_Yes $\underline{X}$

8. Measures of at least one key outcome were obtained from more than $85 \%$ of the subjects initially allocated to groups

9. All subjects for whom outcome measures were available received the No $\underline{X}$ Yes

No $\underline{X}$ Yes treatment or control condition as allocated or, where this was not the case, data for at least one key outcome was analysed by "intention to treat"

10. The results of between-group statistical comparisons are reported for at least one key outcome

11. The study provides both point measures and measures of variability for at No_Yes $\underline{X}$ least one key outcome

Total: $5 / 10$

Table D15. Hoch et al. ${ }^{35}$ PEDro scale

1. Eligibility criteria were specified No_Yes $\underline{\mathrm{X}}$

2. Subjects were randomly allocated to groups (in a crossover study, subjects were randomly allocated an order in which treatments were received)

3. Allocation was concealed

4. The groups were similar at baseline regarding the most important

No $\underline{X}$ Yes prognostic indicators

5. There was blinding of all subjects

6. There was blinding of all therapists who administered the therapy

No $\underline{X}$ Yes

7. There was blinding of all assessors who measured at least one key

No_Yes $\underline{X}$

8. Measures of at least one key outcome were obtained from more than $85 \%$ of the subjects initially allocated to groups

9. All subjects for whom outcome measures were available received the

No $\underline{X}$ Yes No $\underline{X}$ Yes No $\underline{X}$ Yes No_Yes $\underline{X}$ treatment or control condition as allocated or, where this was not the case, data for at least one key outcome was analysed by "intention to treat"

10. The results of between-group statistical comparisons are reported for at $\quad$ No_Yes $\underline{X}$ least one key outcome

11. The study provides both point measures and measures of variability for at No _ Yes $\underline{X}$ least one key outcome 
Table D16. Marrón-Gómez et al.36 PEDro scale

1. Eligibility criteria were specified No $\underline{X}$ Yes

2. Subjects were randomly allocated to groups (in a crossover study, subjects No _ Yes $\underline{X}$ were randomly allocated an order in which treatments were received)

3. Allocation was concealed

No $\underline{X}$ Yes

4. The groups were similar at baseline regarding the most important

No_Yes $\underline{X}$ prognostic indicators

5. There was blinding of all subjects

No_Yes $\underline{X}$

6. There was blinding of all therapists who administered the therapy

No $\underline{X}$ Yes

7. There was blinding of all assessors who measured at least one key

No_Yes $\underline{X}$

8. Measures of at least one key outcome were obtained from more than $85 \%$ of the subjects initially allocated to groups

9. All subjects for whom outcome measures were available received the

No_Yes $\underline{X}$

No $\underline{X}$ Yes treatment or control condition as allocated or, where this was not the case, data for at least one key outcome was analysed by "intention to treat"

10. The results of between-group statistical comparisons are reported for at least one key outcome

11. The study provides both point measures and measures of variability for at No_Yes $\underline{X}$ least one key outcome

Total: $7 / 10$

Table D17. Nambi et al. ${ }^{37}$ PEDro scale

1. Eligibility criteria were specified No_Yes $\underline{\mathrm{X}}$

2. Subjects were randomly allocated to groups (in a crossover study, subjects were randomly allocated an order in which treatments were received)

3. Allocation was concealed

4. The groups were similar at baseline regarding the most important

No $\underline{X}$ Yes prognostic indicators

5. There was blinding of all subjects

6. There was blinding of all therapists who administered the therapy

No $\underline{X}$ Yes

7. There was blinding of all assessors who measured at least one key

No_Yes $\underline{\mathrm{X}}$

8. Measures of at least one key outcome were obtained from more than $85 \%$ of the subjects initially allocated to groups

9. All subjects for whom outcome measures were available received the treatment or control condition as allocated or, where this was not the case, data for at least one key outcome was analysed by "intention to treat"

10. The results of between-group statistical comparisons are reported for at $\quad$ No _ Yes $\underline{X}$ least one key outcome

11. The study provides both point measures and measures of variability for at No _ Yes $\underline{X}$ least one key outcome

No $\underline{X}$ Yes No $\underline{X}$ Yes No $\underline{X}$ Yes No $\underline{X}$ Yes No $\underline{X}$ Yes

Total: 3/10 
Table D18. Pellow et al. ${ }^{38}$ PEDro scale

1. Eligibility criteria were specified $\quad$ No _ Yes $\underline{X}$

2. Subjects were randomly allocated to groups (in a crossover study, subjects No _Yes $\underline{X}$ were randomly allocated an order in which treatments were received)

3. Allocation was concealed

No $\underline{X}$ Yes

4. The groups were similar at baseline regarding the most important

No $\underline{X}$ Yes prognostic indicators

5. There was blinding of all subjects

No $\underline{X} Y e s$

6. There was blinding of all therapists who administered the therapy

No $\underline{X}$ Yes

7. There was blinding of all assessors who measured at least one key

No_Yes $\underline{X}$

8. Measures of at least one key outcome were obtained from more than $85 \%$ of the subjects initially allocated to groups

9. All subjects for whom outcome measures were available received the

No_Yes $\underline{X}$

No_Yes $\underline{X}$ treatment or control condition as allocated or, where this was not the case, data for at least one key outcome was analysed by "intention to treat"

10. The results of between-group statistical comparisons are reported for at least one key outcome

11. The study provides both point measures and measures of variability for at No _ Yes $\underline{X}$ least one key outcome

Total: $5 / 10$

Table D19. Reid et al. ${ }^{39}$ PEDro scale

1. Eligibility criteria were specified No_Yes $\underline{X}$

2. Subjects were randomly allocated to groups (in a crossover study, subjects were randomly allocated an order in which treatments were received)

3. Allocation was concealed

4. The groups were similar at baseline regarding the most important

No $\underline{X}$ Yes prognostic indicators

5. There was blinding of all subjects

6. There was blinding of all therapists who administered the therapy

No_Yes $\underline{\mathrm{X}}$

7. There was blinding of all assessors who measured at least one key

No_Yes $\underline{X}$

8. Measures of at least one key outcome were obtained from more than 85\% of the subjects initially allocated to groups

9. All subjects for whom outcome measures were available received the

No $\underline{X}$ Yes No $\underline{X}$ Yes No_Yes $\underline{\mathrm{X}}$

No_Yes $\underline{X}$ treatment or control condition as allocated or, where this was not the case, data for at least one key outcome was analysed by "intention to treat"

10. The results of between-group statistical comparisons are reported for at $\quad$ No_Yes $\underline{X}$ least one key outcome

11. The study provides both point measures and measures of variability for at No _Yes $\underline{X}$ least one key outcome 
Table D20. Vicenzino et al. ${ }^{40}$ PEDro scale

1. Eligibility criteria were specified $\quad$ No _ Yes $\underline{X}$

2. Subjects were randomly allocated to groups (in a crossover study, subjects No _Yes $\underline{X}$ were randomly allocated an order in which treatments were received)

3. Allocation was concealed

No $\underline{X}$ Yes

4. The groups were similar at baseline regarding the most important

No_Yes $\underline{X}$ prognostic indicators

5. There was blinding of all subjects

No $\underline{X}$ Yes

6. There was blinding of all therapists who administered the therapy

No $\underline{X}$ Yes

7. There was blinding of all assessors who measured at least one key

No_Yes $\underline{X}$

8. Measures of at least one key outcome were obtained from more than $85 \%$ of the subjects initially allocated to groups

9. All subjects for whom outcome measures were available received the No $\underline{X} Y e s$ treatment or control condition as allocated or, where this was not the

No_Yes $\underline{X}$ case, data for at least one key outcome was analysed by "intention to treat"

10. The results of between-group statistical comparisons are reported for at least one key outcome

11. The study provides both point measures and measures of variability for at No_Yes $\underline{X}$ least one key outcome

Total: $6 / 10$

Table D21. Yeo et al. ${ }^{41}$ PEDro scale

1. Eligibility criteria were specified

No _ Yes $\underline{\mathrm{X}}$

2. Subjects were randomly allocated to groups (in a crossover study, subjects were randomly allocated an order in which treatments were received)

3. Allocation was concealed

No_Yes $\underline{X}$

4. The groups were similar at baseline regarding the most important prognostic indicators

5. There was blinding of all subjects

No $\underline{X}$ Yes

No_Yes $\underline{X}$

6. There was blinding of all therapists who administered the therapy

No $\underline{X}$ Yes

No $\underline{X} Y e s$ _

7. There was blinding of all assessors who measured at least one key

No_Yes $\underline{X}$

8. Measures of at least one key outcome were obtained from more than $85 \%$ of

No_Yes $\underline{X}$ the subjects initially allocated to groups

9. All subjects for whom outcome measures were available received the

No $\underline{X} Y e s$ _ treatment or control condition as allocated or, where this was not the case, data for at least one key outcome was analysed by "intention to treat"

10. The results of between-group statistical comparisons are reported for at least No _ Yes $\underline{X}$ one key outcome

11. The study provides both point measures and measures of variability for at $\quad$ No $\underline{X}$ Yes least one key outcome

Total: $5 / 10$ 
Table D22. Effect Size for Andersen et al. ${ }^{27}$

\begin{tabular}{llll}
\hline & Effect Size & $95 \%$ CI: Lower & $95 \%$ CI: Upper \\
$\begin{array}{l}\text { Torque: Pre-treatment vs Post- } \\
\text { treatment }\end{array}$ & 0.6 & -0.48 & 0.6 \\
$\begin{array}{l}\text { Torque: treatment vs control } \\
\begin{array}{l}\text { Dorsiflexion: Pre-treatment vs post- } \\
\text { treatment }\end{array}\end{array}$ & 0 & -0.55 & 0.55 \\
Dorsiflexion: treatment vs control & 0.3 & -0.48 & 0.61 \\
\hline
\end{tabular}

Table D23. Effect Size for Beazell et al. ${ }^{28}$ on Dorsiflexion

\begin{tabular}{llll}
\hline & Effect Size & $95 \%$ CI: Lower & $95 \%$ CI: Upper \\
PTFJM pre vs day 1 & 0.35 & -0.37 & 1.07 \\
PTFJM pre vs day 7 & 0.43 & -0.3 & 1.15 \\
PTFJM pre vs day 14 & 0.54 & -0.19 & 1.27 \\
PTFJM pre vs day 21 & 0.7 & -0.04 & 1.44 \\
DTFJM pre vs day 1 & 0.4 & -0.32 & 1.13 \\
DTFJM pre vs day 7 & 0.6 & -0.13 & 1.33 \\
DTFJM pre vs day 14 & 0.57 & -0.16 & 1.3 \\
DTFJM pre vs day 21 & 0.53 & -0.2 & 1.26 \\
Day 1: PTFJM vs Control & 0.07 & -0.63 & 0.78 \\
Day 1 DTFJM vs Control & -0.08 & -0.79 & 0.62 \\
Day7: PTFJM vs Control & 0.06 & -0.65 & 0.76 \\
Day 7: DTFJM vs Control & -0.03 & -0.73 & 0.68 \\
Day 14: PTFJM vs Control & 0.16 & -0.55 & 0.87 \\
Day 14: DTFJM vs Control & -0.03 & -0.73 & 0.68 \\
Day 21: PTFJM vs Control & 0.12 & -0.59 & 0.82 \\
Day 21: DTFJM vs Control & -0.27 & -0.97 & 0.44 \\
\hline
\end{tabular}


Table D24. Effect Size for Collins et al. ${ }^{29}$

\begin{tabular}{|c|c|c|c|}
\hline & Effect Size & 95\% CI: Lower & 95\% CI: Upper \\
\hline Dorsiflexion: treatment vs placebo & 0.18 & -0.52 & 0.87 \\
\hline Dorsiflexion: treatment vs control & 0.37 & -0.32 & 1.07 \\
\hline $\begin{array}{l}\text { Pain Pressure Threshold (PPT) ATF: } \\
\text { treatment vs placebo }\end{array}$ & -0.16 & -0.86 & 0.53 \\
\hline PPT ATF: treatment vs control & -0.21 & -0.9 & 0.49 \\
\hline PPT CF: treatment vs placebo & -0.04 & -0.74 & 0.76 \\
\hline PPT CF: treatment vs control & 0.07 & -0.63 & 0.76 \\
\hline PPT TA: treatment vs placebo & 0.37 & -0.63 & 0.76 \\
\hline PPT TA: treatment vs control & -0.14 & -0.83 & 0.55 \\
\hline $\begin{array}{l}\text { Heat Threshold to ATF: treatment vs } \\
\text { placebo }\end{array}$ & -0.47 & -1.17 & 0.24 \\
\hline $\begin{array}{l}\text { Heat Threshold to ATF: treatment vs } \\
\text { control }\end{array}$ & -0.46 & -1.16 & 0.25 \\
\hline $\begin{array}{l}\text { Heat threshold to TA: treatment vs } \\
\text { placebo }\end{array}$ & 0.13 & -0.56 & 0.83 \\
\hline $\begin{array}{l}\text { Heat threshold to TA: treatment vs } \\
\text { control }\end{array}$ & -0.11 & -0.81 & 0.58 \\
\hline $\begin{array}{l}\text { Cold Threshold to ATF: treatment vs } \\
\text { placebo }\end{array}$ & -0.14 & -0.83 & 0.56 \\
\hline $\begin{array}{l}\text { Cold Threshold to ATF: treatment vs } \\
\text { control }\end{array}$ & 0.15 & -0.55 & 0.84 \\
\hline $\begin{array}{l}\text { Cold threshold to TA: treatment vs } \\
\text { placebo }\end{array}$ & 0.01 & -0.68 & 0.71 \\
\hline $\begin{array}{l}\text { Cold threshold to TA: treatment vs } \\
\text { control }\end{array}$ & 0.6 & -0.63 & 0.76 \\
\hline
\end{tabular}

Table D25. Effect Size for Cosby et al. ${ }^{30}$

\begin{tabular}{|c|c|c|c|}
\hline & Effect Size & 95\% CI: Lower & 95\% CI: Upper \\
\hline $\begin{array}{l}\text { Dorsiflexion: Pre-treatment vs } \\
\text { Post-treatment }\end{array}$ & 0.02 & -0.9 & 0.9 \\
\hline $\begin{array}{l}\text { Dorsiflexion: Pre-treatment vs } \\
24 \text { hour post-treatment }\end{array}$ & 0.36 & -0.57 & 1.29 \\
\hline $\begin{array}{l}\text { Posterior Glide: Pre-treatment } \\
\text { vs post-treatment }\end{array}$ & -0.06 & -0.99 & 0.94 \\
\hline $\begin{array}{l}\text { Posterior Glide: Pre-treatment } \\
\text { vs post-treatment }\end{array}$ & -0.01 & -0.93 & 0.92 \\
\hline $\begin{array}{l}\text { Dorsiflexion Post-treatment: } \\
\text { Treatment vs Control }\end{array}$ & 0.26 & -0.7 & 1.21 \\
\hline $\begin{array}{l}\text { Posterior Glide Post-treatment: } \\
\text { Treatment vs Control }\end{array}$ & 0.51 & -0.46 & 1.48 \\
\hline $\begin{array}{l}\text { Dorsiflexion } 24 \text { hour post } \\
\text { treatment: Treatment vs } \\
\text { Control }\end{array}$ & -0.28 & -1.24 & 0.68 \\
\hline $\begin{array}{l}\text { Posterior Glide } 24 \text { hour Post- } \\
\text { treatment: Treatment vs } \\
\text { Control }\end{array}$ & 0.03 & -0.92 & 0.98 \\
\hline
\end{tabular}


Table D26. Effect Size for Gilbreath et al. ${ }^{31}$

\begin{tabular}{llll}
\hline & Effect Size & $95 \%$ CI: Lower & 95\% CI: Upper \\
$\begin{array}{l}\text { Dorsiflexion: pre-treatment vs post- } \\
\text { treatment }\end{array}$ & 0.16 & -0.68 & 1 \\
\hline
\end{tabular}

Table D27. Effect Size for Hoch et al. ${ }^{34}$

\begin{tabular}{llll}
\hline & Effect Size & $95 \%$ CI: Lower & $95 \%$ CI: Upper \\
$\begin{array}{l}\text { Dorsiflexion: Treatment vs control } \\
\begin{array}{l}\text { Posterior Talar displacement: } \\
\text { treatment vs control }\end{array}\end{array}$ & 0.14 & -0.48 & 0.76 \\
$\begin{array}{l}\text { Posterior Stiffness: treatment vs } \\
\text { control }\end{array}$ & 0.37 & -0.26 & 0.99 \\
\hline
\end{tabular}

Table D28. Effect Size for Hoch et al. ${ }^{35}$

\begin{tabular}{llll}
\hline & Effect Size & $95 \%$ CI: Lower & $95 \%$ CI: Upper \\
$\begin{array}{l}\text { Dorsiflexion: Pre-treatment vs Post- } \\
\text { treatment }\end{array}$ & 0.35 & -0.45 & 1.16 \\
$\begin{array}{l}\text { Dorsiflexion: Pre-treatment vs 1 week } \\
\text { follow up }\end{array}$ & 0.38 & -0.42 & 1.14 \\
\hline
\end{tabular}

Table D29. Effect Size for Marrón-Gómez et al. ${ }^{36}$

Effect Size $\quad 95 \%$ CI: Lower $\quad 95 \%$ CI: Upper

\begin{tabular}{llll} 
HVLA pre/post test & 0.6 & -0.5 & 1.25 \\
HVLA pre to 10 min after & 0.73 & 0.7 & 1.39 \\
HVLA pre to 24 hr after & 0.7 & 0.05 & 1.36 \\
HVLA pre to 48 hr after & 0.47 & -0.18 & 1.11 \\
MWM pre/post & 0.48 & -0.18 & 1.15 \\
MWM pre to 10 min after & 0.57 & -0.1 & 1.24 \\
MWM pre to 24 hr after & 0.6 & -0.07 & 1.27 \\
MWM pre to 48 hr after & 0.63 & -0.4 & 1.3 \\
HVLA to placebo immediate & 3.4 & 2.35 & 4.45 \\
HVLA to placebo 10 min after & 3.93 & 2.78 & 5.08 \\
HVLA to placebo 24 hr after & 3.86 & 2.72 & 5 \\
HVLA to placebo 48 hr after & 3.07 & 2.07 & 4.06 \\
MWM vs placebo immediate after & 0.48 & -0.18 & 1.15 \\
MWM vs placebo 10 min after & 0.57 & -0.1 & 1.24 \\
MWM vs placebo 24 hr after & 0.6 & -0.7 & 1.27 \\
MWM vs placebo $48 \mathrm{hr}$ after & 0.6 & -0.4 & 1.3 \\
\hline
\end{tabular}

Red indicates an effect size that did not encompass 0 
Table D30. Effect Size for Nambi et al. ${ }^{37}$

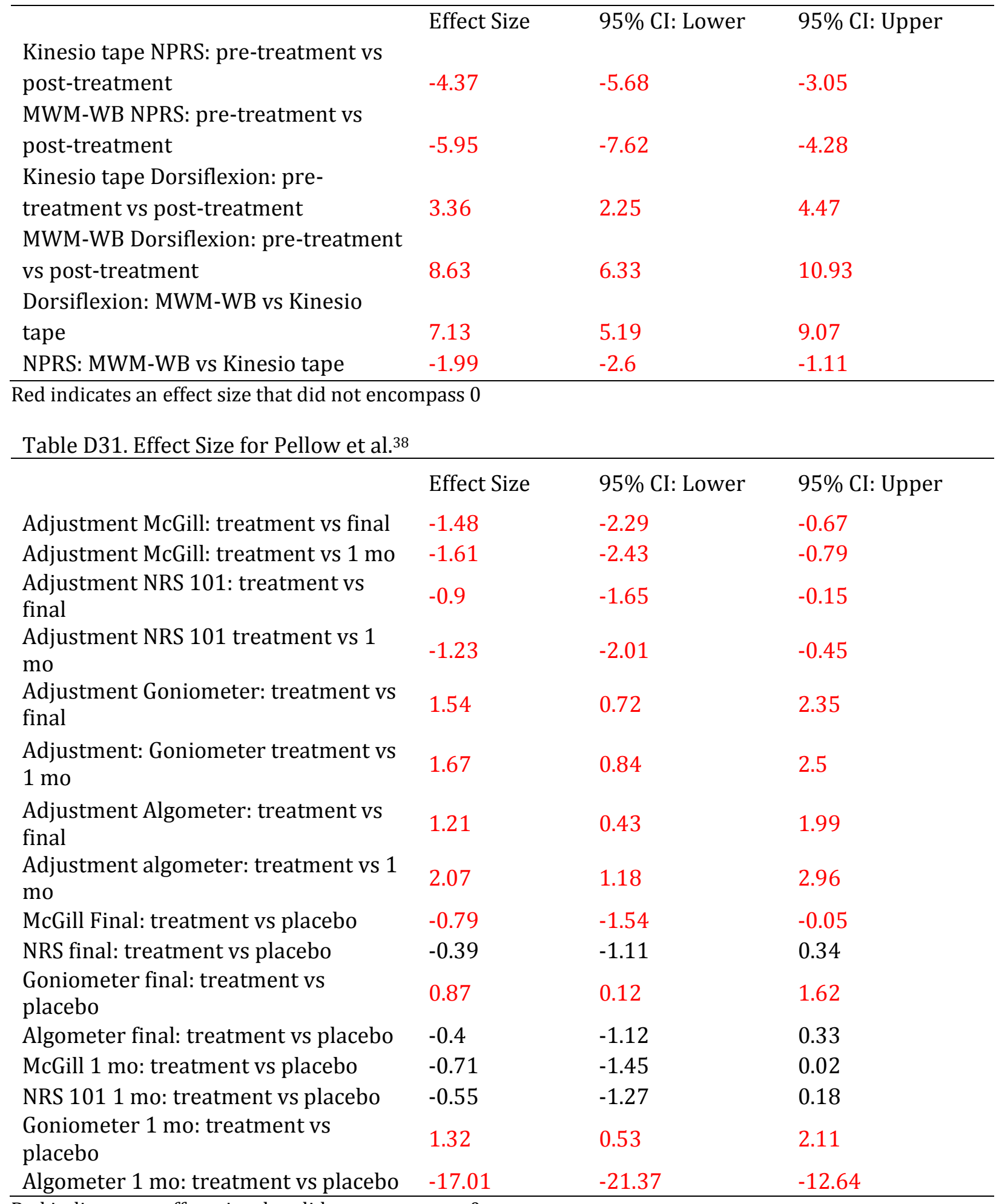

Red indicates an effect size that did not encompass 0 
Table D32. Effect Size for Vicenzino et al. ${ }^{40}$

\begin{tabular}{|c|c|c|c|}
\hline & Effect Size & 95\% CI: Lower & 95\% CI: Upper \\
\hline $\begin{array}{l}\text { Dorsiflexion Pre/Post Treatment: } \\
\text { MWM-WB }\end{array}$ & 0.38 & -0.32 & 1.07 \\
\hline $\begin{array}{l}\text { Dorsiflexion Pre/Post Treatment: } \\
\text { MWM-NWB }\end{array}$ & 0.26 & -0.43 & 0.96 \\
\hline Dorsiflexion: MWM-WB vs control & 0.25 & -0.45 & 0.95 \\
\hline Dorsiflexion: MWM-NWB vs control & 0.25 & -0.45 & 0.95 \\
\hline $\begin{array}{l}\text { Posterior Glide Pre/Post Treatment: } \\
\text { MWM-WB }\end{array}$ & 0.74 & 0.2 & 1.46 \\
\hline $\begin{array}{l}\text { Posterior Glide Pre/Post Treatment: } \\
\text { MWM-NWB }\end{array}$ & 0.85 & 0.13 & 1.57 \\
\hline Posterior Glide: MWM-WB vs control & 0.25 & -0.45 & 0.95 \\
\hline Posterior Glide: MWM-NWB vs control & 0.25 & -0.45 & 0.95 \\
\hline
\end{tabular}

Red indicates an effect size that did not encompass 0 
Figure D1. Figure of studies Found: PubMed
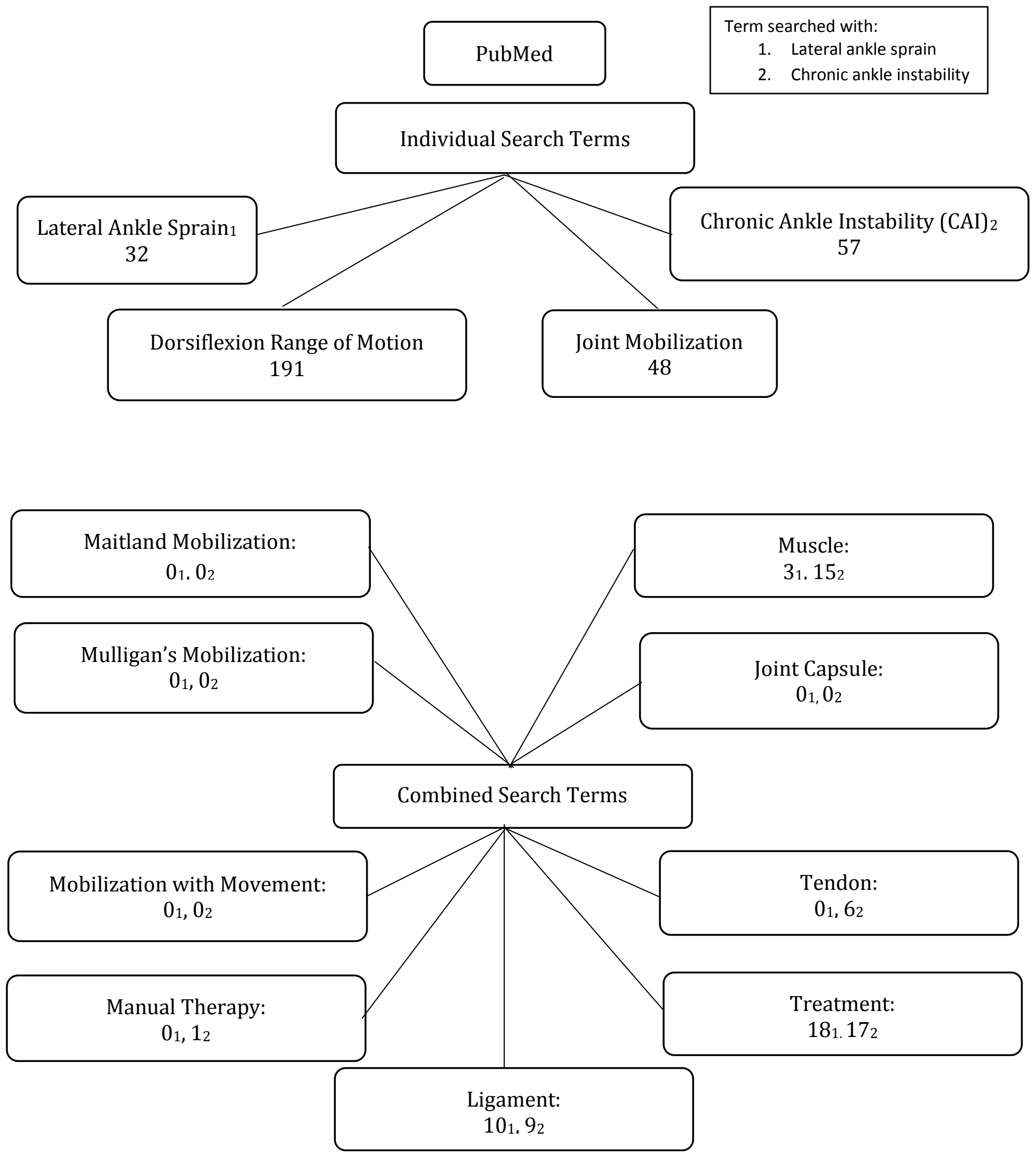
Figure D2. Figure of Studies Found: CINAHL
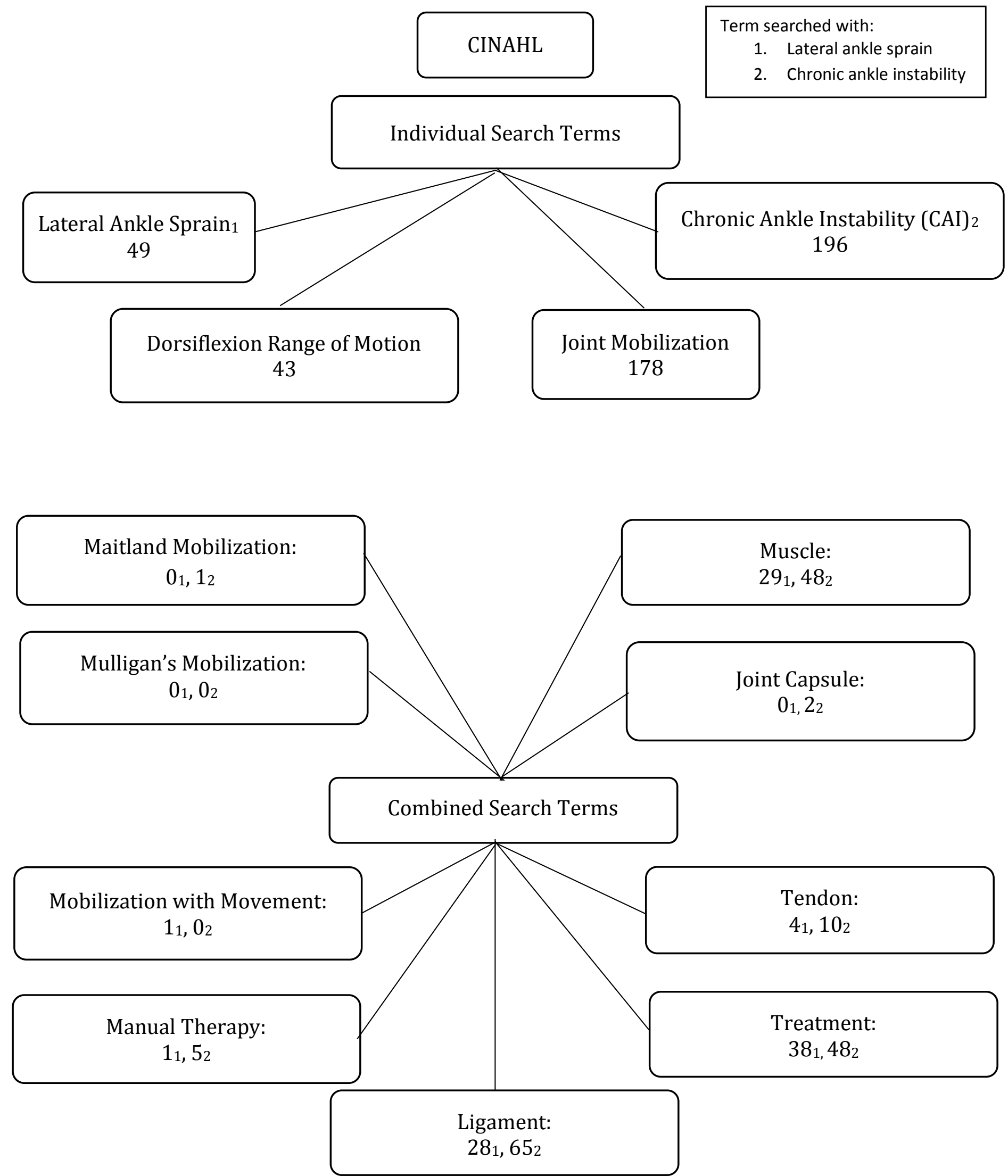
Figure D3. Figure of Studies Found: MEDLINE
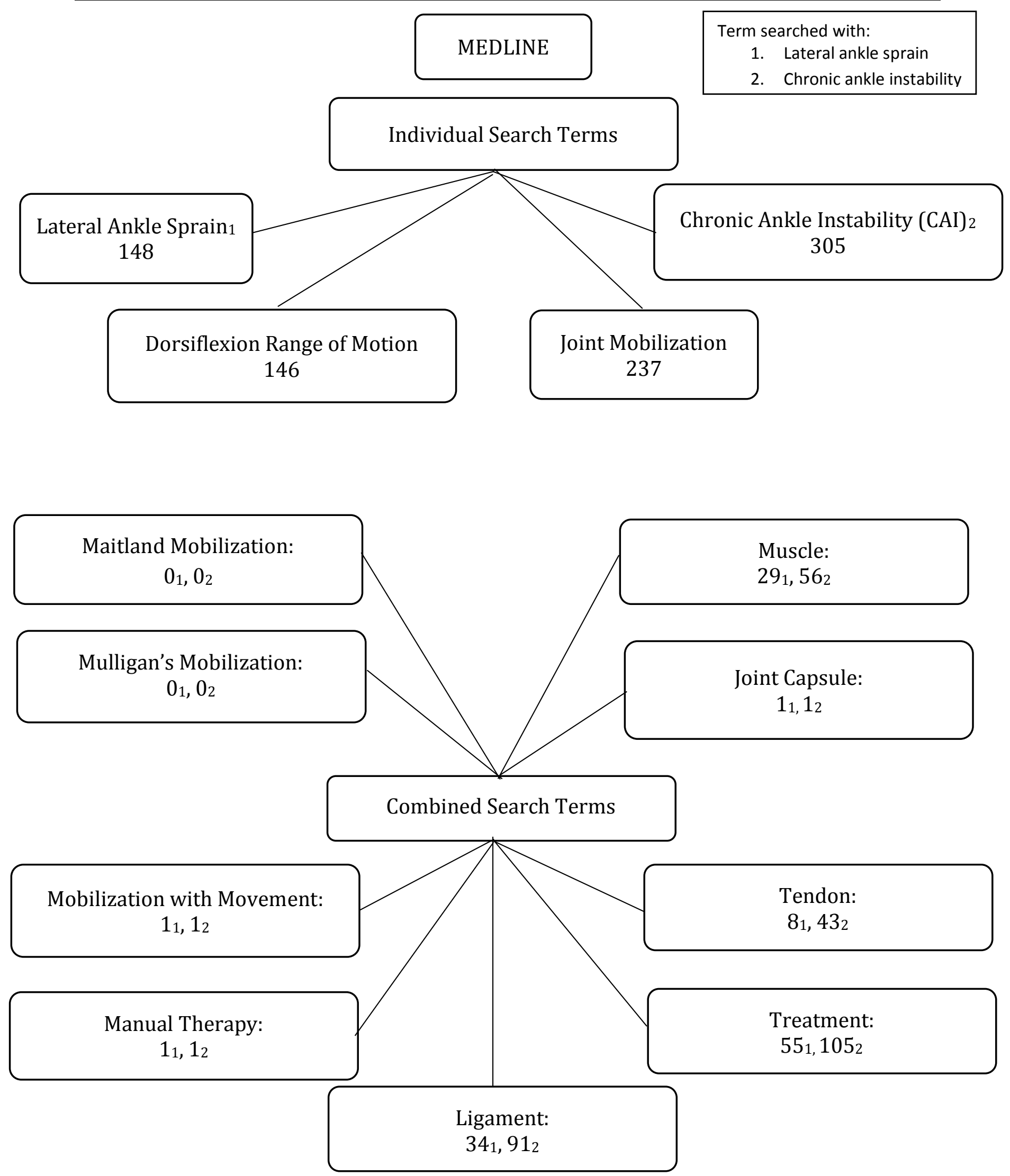

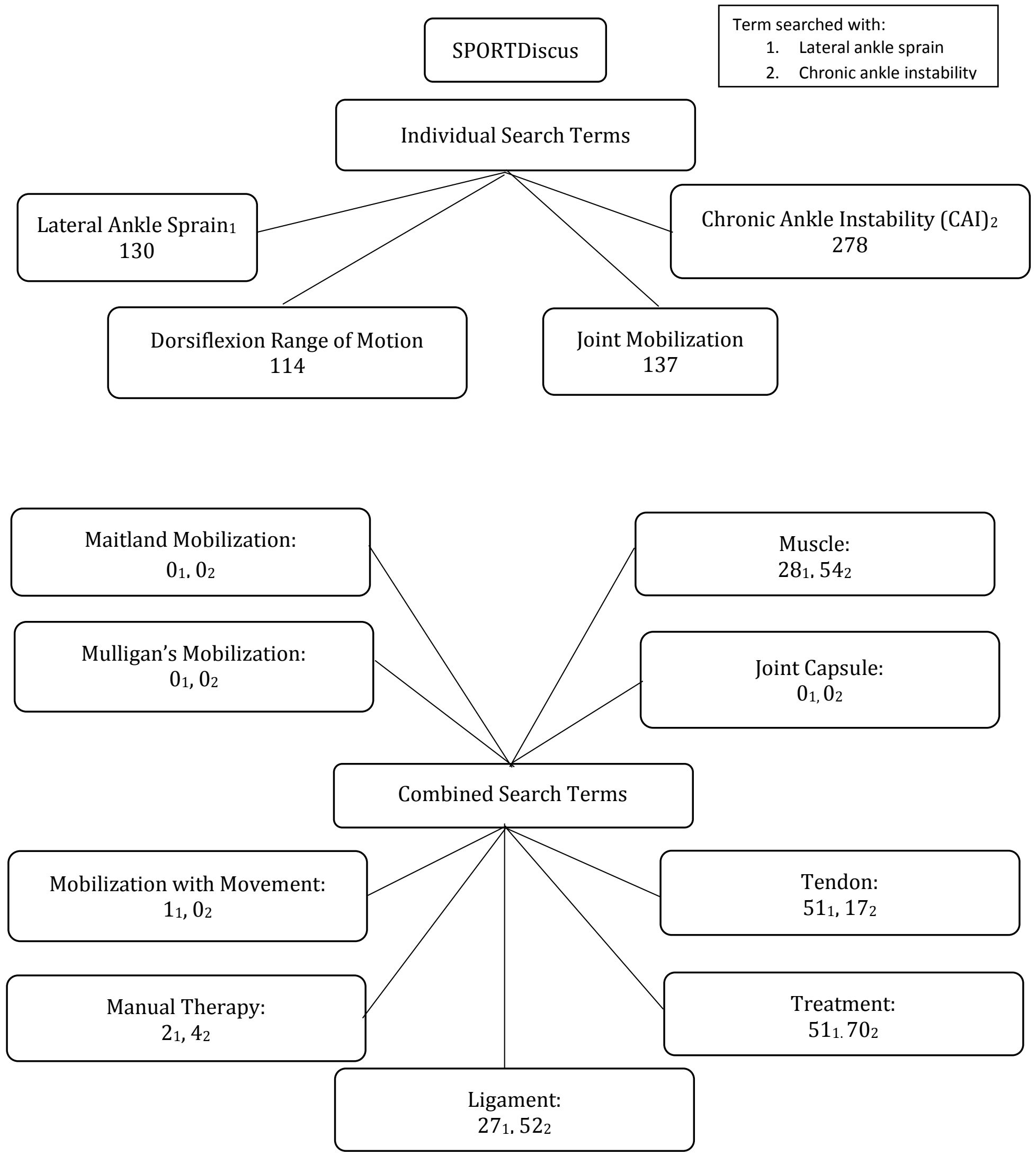
Figure D5. Figure of Studies Found: Science Direct
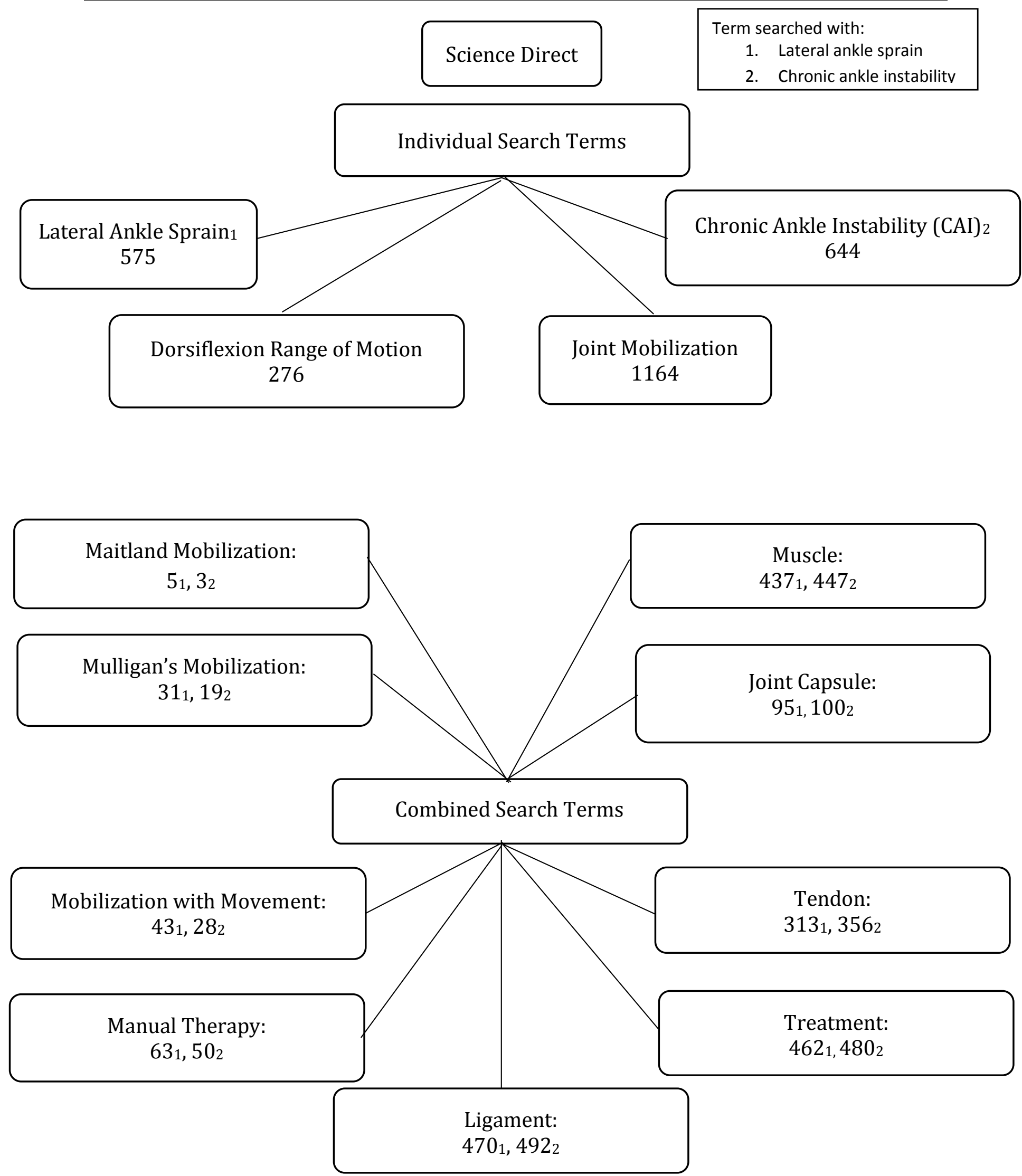
Figure D6. Figure of Studies Found: Google Scholar

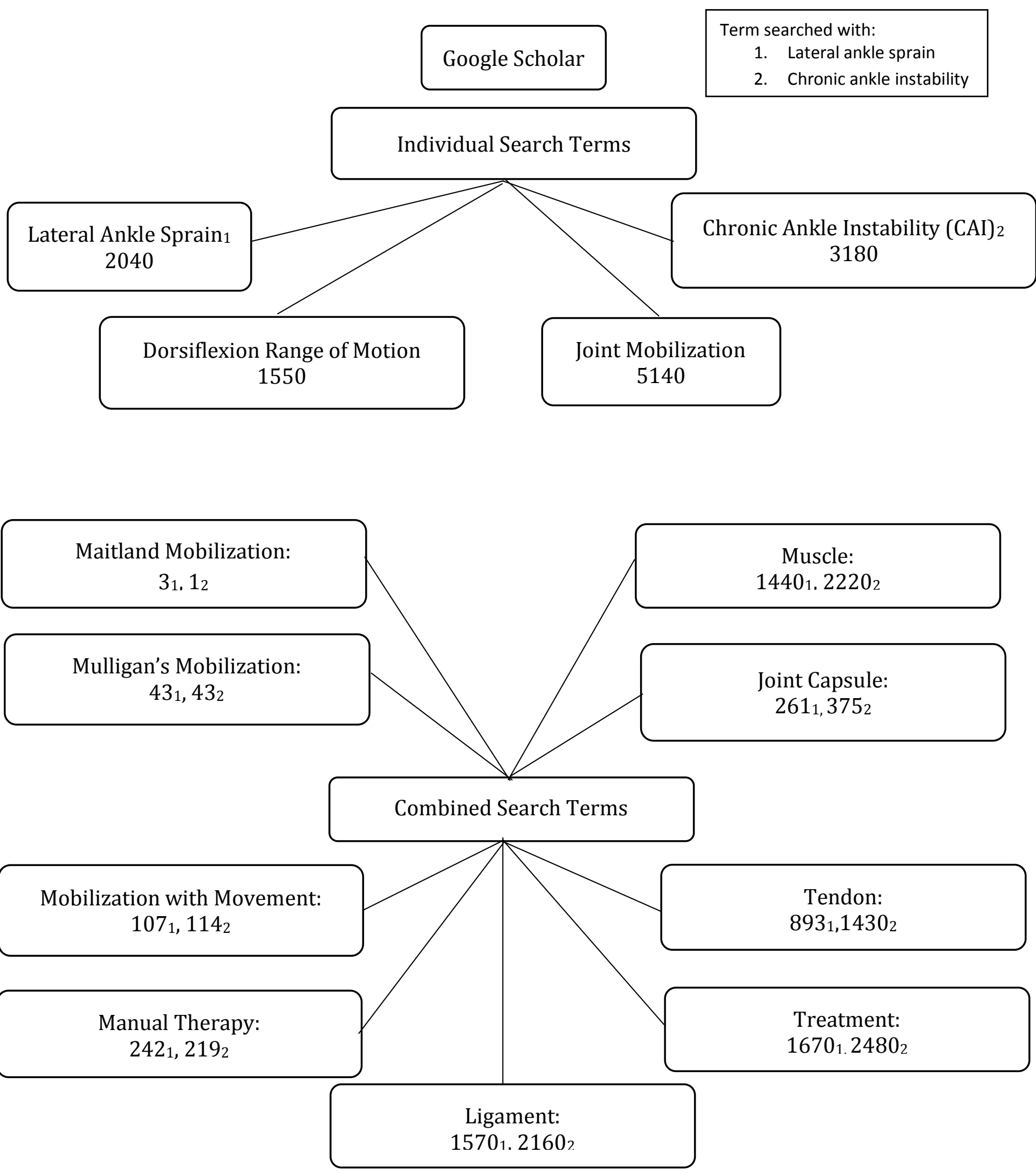



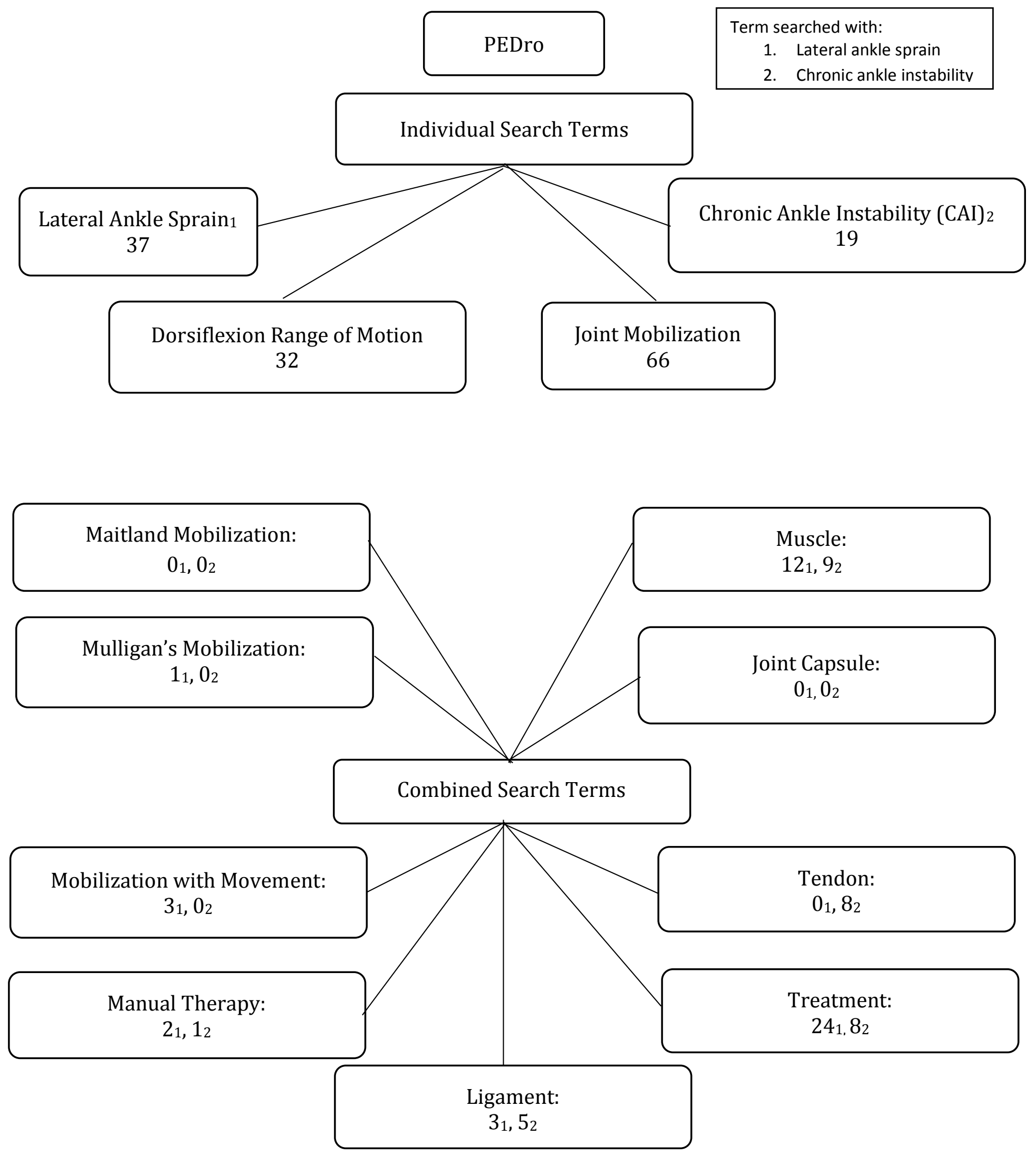
Figure D8. Figure of Studies Included

Search Terms: Primary Terms (lateral ankle sprain, chronic ankle instability (CAI), joint mobilization, and dorsiflexion range of motion) Secondary Terms: lateral ankle sprain or CAI AND the following: Maitland mobilization, Mulligan's mobilization, mobilization with movement, manual therapy, ligament, muscle, joint capsule, tendon, and treatment

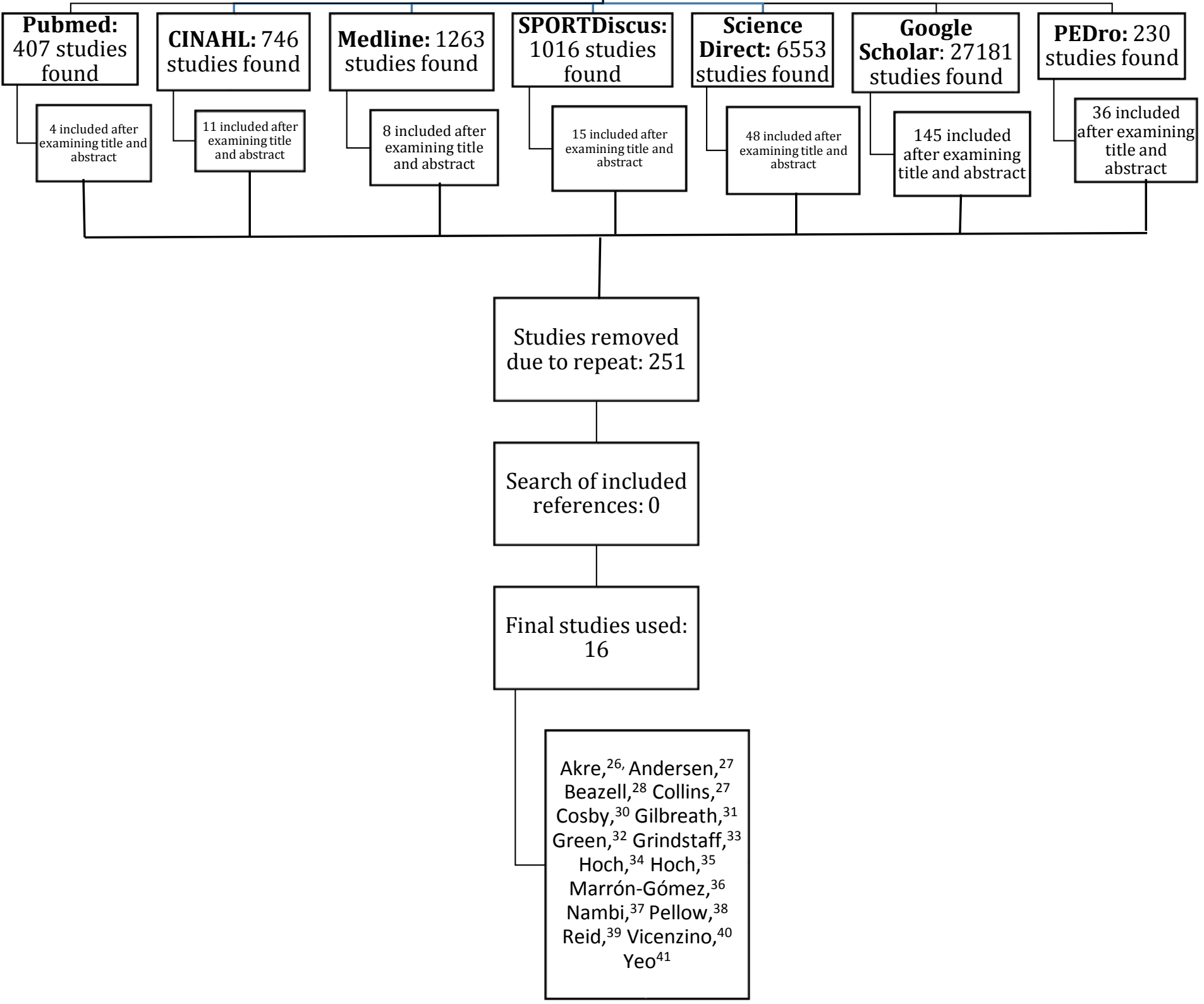




\section{APPENDIX E}

\section{RECOMMENDATIONS FOR FURTHER RESEARCH}

1. Future studies should include more databases and studies written in a foreign language.

2. Studies should work on increasing the methodological quality such as patient blinding, therapist blinding, and concealing of allocation.

3. Expand the inclusion criteria to include other ankle injuries.

4. Studies should focus on multiple treatment sessions and joint mobilizations with traditional treatment as that is what is typically performed in clinical practice.

5. Long term follow-ups would also be beneficial to evaluate if this treatment assisted in reducing re-injury rates. 


\section{ADDITIONAL REFERENCES}

45. Waterman BR, Belmont PJ, Cameron KL, DeBerardio TM, Owens BD. Epidemiology of Ankle Sprain at the United States Military Academy. Am J Sports Medic. 2010; 38:797-803.

46. Petersen W, Rembitzki IV, Koppenburg AG, Ellermann A, Liebau C, Brüggemann GP, Best R. Treatment of acute ankle ligament injuries: a systematic review. Arch Orthop Trauma Surg. 2013; 133:1129-1141.

47. Houglum PA. Therapeutic Exercise for musculoskeletal injures. Champaign, IL: Human Kinetics; 2010; 176-183.

48. De Noronha M, França C, Haupenthal A, Nunes GS. Intrinsic predictive factors for ankle sprain in active university students: a prospective study. Scand J Sci Sports; 2013;23:541-547.

49. Youdas JW, McLean TJ, Krause DA, Hollman JH. Changes in active ankle dorsiflexion range of motion after acute inversion ankle sprain. J Sport Rehabil. 2009;18:358374.

50. Van den Bekerom MP, Struijs PA, Blankevoort L, Welling L, van Dijk N, Kerkhoffs GM. What is the evidence for rest, ice, compression, and elevation therapy in the treatment of ankle sprains in adults. J Athl Train. 2012; 47(4): 435-443.

51. Terada M, Pietrosimone BG, and Gribble PA. Therapeutic interventions for increasing ankle dorsiflexion after ankle sprain: a systematic review. J Athl Train. 2013;48(5):696-709

52. Collado H, Coudreuse JM, Graziani F, Bensoussan L, Viton JM, Delarque A. Eccentric reinforcement of the ankle evertor muscles after ankle sprain. Scand J Med Sci Sports. 2010; 20:241-246.

53. Teixeira LM, Pires T, Silva RD, de Resende MA. Immediate effect of a single anterioposterior talus mobilization on dorsiflexion range of motion in participants with orthopedic dysfunction of the ankle and foot. J Manipulative Physiol Ther. 2013; 36(6):369-375.

54. Houston MN, McKeon PO, Hoch MC. Foot and ankle ability measure scores in patients with chronic ankle instability following joint mobilization. Int J Athl Ther Training. 2013; March: 4-7. 
55. McLean S, Naish R, Reed L, Urry S, Vicenzino B. A pilot study of manual force levels required to produce manipulation induced hypoalgesia. Clin Biomech. 2002;17:304308. 